\title{
Irreversible investment with fixed adjustment costs: a stochastic impulse control approach
}

\author{
Salvatore Federico* $^{*} \quad$ Mauro Rosestolato $^{\dagger} \quad$ Elisa Tacconi $^{\ddagger}$
}

February 5, 2019

\begin{abstract}
We consider an optimal stochastic impulse control problem over an infinite time horizon motivated by a model of irreversible investment choices with fixed adjustment costs. By employing techniques of viscosity solutions and relying on semiconvexity arguments, we prove that the value function is a classical solution to the associated quasi-variational inequality. This enables us to characterize the structure of the continuation and action regions and construct an optimal control. Finally, we focus on the linear case, discussing, by a numerical analysis, the sensitivity of the solution with respect to the relevant parameters of the problem.
\end{abstract}

Keywords: Impulse stochastic optimal control, Quasi-variational inequality, Viscosity solution, Irreversible investment, Fixed cost.

A.M.S. Subject Classification: 93E20 (Optimal stochastic control); 35Q93 (PDEs in connecton woth control and optimization); 35D40 (Viscosity solution); 35B65 (Smoothness and regularity of solutions).

J.E.L. Subject Classification: C61 (Optimization Techniques; Programming Models; Dynamic Analysis); D25 (Intertemporal Firm Choice: Investment, Capacity, and Financing); E22 (Investment; Capital; Intangible Capital; Capacity).

Acknowledgements. The authors are sincerely grateful to the Associate Editor and to two anonymous Referees for their careful reading and very valuable comment that improved the final version of the paper. They also thank Giorgio Ferrari for his very valuable comments and suggestions. Mauro Rosestolato thanks the Department of Political Economics and Statistics of the University of Siena for the kind hospitality in March 2017 and the grant Young Investigator Training Program financed by Associazione di Fondazioni e Casse di Risparmio Spa supporting this visit. He also thanks the ERC 321111 Rofirm for the financial support.

\section{Contents}

\section{Introduction}

\footnotetext{
* Dip. di Economia Politica e Statistica, Università di Siena (Italy). E-mail: salvatore federico@unisi.it.

$\dagger$ CMAP, Ecole Polytechnique, Paris (France). E-mail: mauro.rosestolato@gmail.com.

$¥$ Dipartimento di Finanza, Bocconi University, Milan (Italy). E-mail: elisa.tacconi@unibocconi.it.
} 
3 Preliminary results on the value function $\quad 7$

4 Dynamic Programming 11

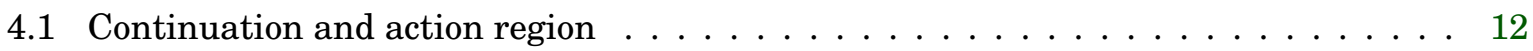

4.2 Dynamic Programming Principle and viscosity solutions . . . . . . . . . . . . . . 14

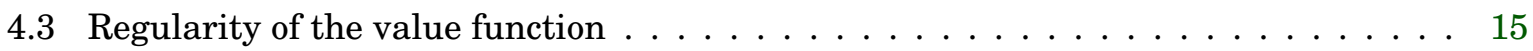

5 Explicit expression of the value function $\quad 16$

6 Optimal control $\quad 24$

7 Numerical illustration in the linear case $\quad 27$

7.1 Numerical illustration . . . . . . . . . . . . . . . . . . . . . . . . . . 29

7.1 .1 Impact of volatility $\ldots \ldots \ldots \ldots \ldots \ldots$

7.1 .2 Impact of fixed cost $\ldots \ldots \ldots \ldots$. . . . . . . . . . . . . .

A Appendix

\section{Introduction}

In this paper we consider a one dimensional stochastic impulse optimal control problem modeling the economic problem of irreversible investment with fixed adjustment cost.

Let $X=\left\{X_{t}\right\}_{t \geq 0}$ be a real valued positive process representing an economic indicator (such as the GDP of a country, the production capacity of a firm, and so on) on which a planner/manager can intervene. When no intervention is undertaken, it is assumed that the process $X$ evolves autonomously according to a time-homogeneous Itô diffusion. On the other hand, the planner may act on this process, increasing its value, by choosing a sequence of interventions dates $\left\{\tau_{n}\right\}_{n \geq 1}$ and of intervention amplitudes $\left\{i_{n}\right\}_{n \geq 1}$, with $i_{n}>0\left(^{1}\right)$. Hence, the control is represented by a sequence of couples $\left\{\left(\tau_{n}, i_{n}\right)\right\}_{n \geq 1}$ : the first component represents the intervention time, the second component the size of intervention. The goal of the controller is to maximize over the set of all admissible controls, the expected total discounted income

$$
\mathbb{E}\left[\int_{0}^{\infty} e^{-\rho t} f\left(X_{t}\right) d t-\sum_{n \geq 1} e^{-\rho \tau_{n}}\left(c_{0} i_{n}+c_{1}\right)\right],
$$

where $f$ is a reward function, $c_{0}>0$ and $c_{1}>0$ represent, respectively, the proportional and the fixed cost of intervention, and $\rho>0$ is a discount factor.

From the modeling side, our problem is the "extension" to the case $c_{1}>0$ of the same problem already treated in the literature in the case $c_{1}=0$ (see, e.g., [63, Ch. 4, Sec. 5]. In this respect, it applies to economic problems of capacity expansion, notably irreversible investment problems $\left({ }^{2}\right)$.

From the theoretical side, the introduction of a fixed cost of control is relevant, as it leads from a problem well posed (in the sense of existence of optimal controls) as a singular control problem

\footnotetext{
${ }^{1}$ The fact that only positive intervention, i.e. $i_{n}>0$, is allowed is expressed in the economic literature of Real Options by saying that the investment is irreversible.

${ }^{2}$ Other than in [63, Ch. 4, Sec. 5], irreversible and reversible investment problems with no fixed investment costs are largely treated in the mathematical economic literature, both over finite and infinite horizon. We mention, among others, [1, 2, 4, 5, 10, 11, 24, 23, 28, 37, 40, 42, 32, 33, 38, 41, 53, 55, 59, 64, 70].
} 
to a problem well posed as an impulse control problem $\left({ }^{3}\right)$. Such a change is not priceless at the theoretical level. Indeed, the introduction of a fixed cost of control has two unpleasant effects. Firstly, it destroys the concavity of the objective functional even if the revenue function is concave. Secondly, when approaching the problem by dynamic programming techniques (as we do), the dynamic programming equation has a nonlocal term and takes the form of a quasi-variational inequality (QVI, hereafter), whereas it is a variational inequality in the singular control case.

Related literature. First of all, it is worth noticing that the stochastic impulse control setting has been widely employed in several applied fields: e.g., exchange and interest rates [21, 51, 56], portfolio optimization with transaction costs [34, 49, 57], inventory and cash management [12, 20, 27, 30, 31, 44, 45, 58, 62, 67, 68, 71], real options [47, 54], reliability theory [7]. More recently, games of stochastic impulse control have been investigated with application to pollution [39].

From a modeling point of view, the closest works to ours can be considered [3, 6, 26, 35, 49]. On the theoretical side, starting from the classical book [17], several works investigated QVIs associated to stochastic impulse optimal control in $\mathbb{R}^{n}$. Among them, we mention the recent [43] in a diffusion setting and [14, 29] in a jump-diffusion setting. In particular, [17, Ch. 4] deals with Sobolev type solutions, whereas [43] deals with viscosity solutions. These two works prove a $W^{2, p}$ regularity, with $p<\infty$, for the solution of QVI, which, by classical Sobolev embeddings, yields a $C^{1}$-regularity. However, it is typically not easy to obtain by such regularity information on the structure of the so called continuation and action regions, hence on the candidate optimal control. If this structure is established, then one can try to prove a verificiation theorem to prove that the candidate optimal control is actually optimal. In a stylized one dimensional example, [43, Sec. 5] successfully employs this method by exploiting the regularity result proved in [43, Sec. 4] to depict the structure of the continuation and action region for the problem at hand. Concerning verification, we need to mention the recent paper [15], which provides a non-smooth verification theorem in a quite general setting based on the stochastic Perron method to construct a viscosity solution to QVI; also this paper, in the last section, provides and application of the results to a one dimensional problem with an implementable solution. In dimension one other approaches, based on excessive mappings and iterated optimal stopping schemes, have been successfully employed in the context of stochastic impulse control (see [3, 6, 35, 46]). More recently, these methods have been extended to Markov processes valued in metric spaces (see [25]); again a complete description of the solution is shown in one dimensional examples.

Contribution. From the methodological side our work is close to [43]. As in the latter, we follow a direct analytical method based on viscosity solutions and we do not employ a guess-and-verify approach $\left({ }^{4}\right)$. Indeed, we directly provide necessary optimality conditions that, by uniqueness, fully characterize the solution. In particular, we do not postulate the smooth-fit principle, as it is usually done in the guess-and-verify approach, but we prove it directly $\left({ }^{5}\right.$.). To the best of our knowledge a rigorous analytical treatment as ours of the specific problem treated in this paper seems to be still missing in the literature. It is important to notice that our analysis yields a a complete and

\footnotetext{
${ }^{3}$ The stochastic impulse control setting has been widely employed in several other applied fields: e.g., exchange rate [21, 51], portfolio optimization with transaction costs [49, 57], inventory and cash management [27, 67, 68], and real options [47, 54].

${ }^{4}$ See, e.g., [13, 27, 49, 50, 57] and, in a much more general context of jump-diffusion, [60, Ch. 6] for the guess-andverify approach.

${ }^{5}$ The smooth-fit principle has also been established, when the diffusion is assumed to be transient, by techniques based on excessive function (see [66])
} 
implementable characterization of the optimal control policy through the identification of the continuation and action regions. Since the aforementioned techniques based on excessive mappings seems to be perfectly employable to our problem (even under weaker assumption), it is worth to point out that our contribution is methodological. As it is well known, the (implementable) characterization of the optimal control in stochastic impulse control problems is a challenging task in dimension larger than one. Hence, it is important to have at hand an approach like ours that might be generalized to address impulse control problems in multi-dimensional setting. To this regard, it is worth to notice the following.

- To the best of our knowledge, the only study providing a complete picture of the solution in dimension two - through a two dimensional $(S, s)$-rule — is the recent paper [16]. The techniques used there are analytical and based on the study of QVI's. Unfortunately, in this paper, the authors are able to provide a complete solution only in a very specific case.

- In the presence of semiconvex data, our approach to prove $C^{1}$ regularity of the value function based on semiconvexity jointly with the viscosity property, unlike [43], might be successful to prove a directional regularity result just along nondegenerate directions (see [37] in a singular control context).

- The directional regularity result mentioned above might be sufficient to derive the right optimality condition to solve the control problem (see again [37] in a singular control context).

Contents. In Section 2 we set up the problem. In Section 3 we state some preliminary results on the value function $v$, in particular we show that it is semiconvex. In Section 4 we derive QVI associated to $v$ and show that it solves the latter in viscosity sense. After that, we prove that $v$ is of class $C^{2}$ in the continuation region (the region where the differential part of QVI holds with equality, see below) and of class $C^{1}$ on the whole state space (Theorem 4.6, our first main result), hence proving the smooth fit-principle. We prove the latter result relying just on the semiconvexity of $v$ and exploting the viscosity supersolution property; unlike [43], this allows to avoid the use of a deep theoretical result such as the Calderon-Zygmund estimate. So, with respect to the aforementioned reference, our method of proof is cheaper from a theoretical point of view; on the other hand, it heavily relies on assumptions guaranteeing the semiconvexity of $v$. In Section 5 we use the latter regularity to establish the structure of the continuation and action regions - the real unknown of the problem - showing that they are both intervals. This allows to express explicitly $v$ up to the solution of a nonlinear algebraic system of three variables (Theorem 5.11 , our second main result). In Section 6, relying on the results of the previous section, we are able to construct an optimal control policy (Theorem 6.1, our third main result). The latter turns out to be based on the so called $(S, s)$-rule $\left({ }^{6}\right)$ : the controller acts whenever the state process reaches a minimum level $s$ (the "trigger" boundary) and brings immediately the system at the level $S>s$ (the "target" boundary). Finally, in Section 7, we provide a numerical illustration of the solution when $X$ follows a geometric Brownian motion dynamics between intervation times, analyzing the sensitivity of the solution with respect to the volatility coefficient $\sigma$ and to and the fixed $\operatorname{cost} c_{1}$.

\footnotetext{
${ }^{6}$ This is a well known rule in the economic literature of inventory problems, see [8, 67, 68].
} 


\section{Problem formulation}

We introduce some notation. We set

$$
\mathbb{R}_{+}:=[0,+\infty), \quad \overline{\mathbb{R}}_{+}:=[0,+\infty], \quad \mathbb{R}_{++}:=(0,+\infty) .
$$

The set $\mathbb{R}_{++}$will be the state space of our control problem. Throughout the paper we adopt the conventions $e^{-\infty}=0$ and $\inf \varnothing=\infty$. Moreover, we simply use the symbol $\infty$ in place of $+\infty$ when positive quantities are involved and no confusion may arise. Finally, the symbol $n$ will always denote a natural number.

Let $\left(\Omega, \mathscr{F},\left\{\mathscr{F}_{t}\right\}_{t \geq 0}, \mathbb{P}\right)$ be a filtered probability space satisfying the usual conditions and supporting a a one dimensional Brownian motion $W=\left\{W_{t}\right\}_{t \geq 0}$. We denote $\mathbb{F}:=\left\{\mathscr{F}_{t}\right\}_{t \in \overline{\mathbb{R}}_{+}}$, where we set $\mathscr{F}_{\infty}:=\bigvee_{t \in \mathbb{R}_{+}} \mathscr{F}_{t}$. We take $b, \sigma: \mathbb{R} \rightarrow \mathbb{R}$ satisfying the following

Assumption 2.1. $b, \sigma: \mathbb{R} \rightarrow \mathbb{R}$ are Lipschitz continuous functions, with Lipschitz constants $L_{b}, L_{\sigma}$, respectively, identically equal to 0 on $(-\infty, 0]$, and with $\sigma>0$ on $\mathbb{R}_{++}$. Moreover, $b, \sigma \in C^{1}\left(\mathbb{R}_{+}\right)$, and $b^{\prime}, \sigma^{\prime}$ are Lipschitz continuous on $\mathbb{R}_{++}$, with Lipschitz constants $\tilde{L}_{b}, \tilde{L}_{\sigma}>0$, respectively.

Remark 2.2. The requirement that $b^{\prime}, \sigma^{\prime}$ are Lipschitz continuous is typical when one wants to prove the semiconvexity/semiconcavity of the value function in stochastic optimal control problem (see, e.g., the classical reference [72, Ch. 4, Sec. 4.2] in the context of regular stochastic control; [14] in the context of impulse control). We use this assumpton since, as outlined in the introduction, in our approach the proof of the semiconvexity of the value function will be a crucial step towards the proof of the $C^{1}$ regularity.

Let $\tau$ be a (possibly not finite) $\mathbb{F}$-stopping time and let $\xi$ be an $\mathscr{F}_{\tau}$-measurable random variable. By standard SDE's theory with Lipschitz coefficients, Assumption 2.1 guarantees that there exists a unique (up to undistinguishability) $\mathbb{F}$-adapted process $Z^{\tau, \xi}=\left\{Z_{t}^{\tau, \xi}\right\}_{t \geq 0}$ with continuous trajectories on $[\tau, \infty)$, such that

$$
Z_{t}^{\tau, \xi}= \begin{cases}0 & \text { for } t \in[0, \tau) \\ \xi+\int_{\tau}^{t} b\left(Z_{s}^{\tau, \xi \xi}\right) d s+\int_{\tau}^{t} \sigma\left(Z_{s}^{\tau, \xi}\right) d W_{s} & \mathbb{P} \text {-a.s., for } t \geq \tau .\end{cases}
$$

Moreover, by a straightforward adaptation of [48, Sec. 5.2, Prop. 2.18] to random initial data, we obtain

$$
\xi, \eta \mathscr{F}_{\tau} \text {-measurable random variables, } \xi \leq \eta \mathbb{P} \text {-a.s. } \Longrightarrow Z_{t+\tau}^{\tau, \xi} \leq Z_{t+\tau}^{\tau, \eta} \mathbb{P} \text {-a.s., } \forall t \geq 0 .
$$

Now fix $x \in \mathbb{R}_{++}$. By (2.2) and Assumption 2.1, it follows that $Z^{0, x}$ takes values in $\mathbb{R}_{+}$. Due to the nondegeneracy assumption on $\sigma$ over $\mathbb{R}_{++}$, as a consequence of the results of [48, Sec. 5.5.C], the process $Z^{0, x}$ is a (time-homogeneous) regular diffusion on $\mathbb{R}_{++}$; i.e., setting $\tau_{x, y}:=\inf \left\{t \geq 0: Z_{t}^{0, x}=y\right\}$, one has

$$
\mathbb{P}\left\{\tau_{x, y}<\infty\right\}>0 \quad \forall y \in \mathbb{R}_{++} .
$$

In Appendix we show that Assumption 2.1 guarantees that the boundaries 0 and $+\infty$ are natural for $Z^{0, x}$ in the sense of Feller's classification.

We introduce now a set of admissible controls and their corresponding controlled process. As a set of admissible controls (i.e., feasible investment strategies) we consider the set $\mathscr{I}$ of all sequences of couples $I=\left\{\left(\tau_{n}, i_{n}\right)\right\}_{n \geq 1}$ such that: 
(i) $\left\{\tau_{n}\right\}_{n \geq 1}$ is an increasing sequence of $\overline{\mathbb{R}}_{+}$-valued $\mathbb{F}$-stopping times such that $\tau_{n}<\tau_{n+1} \mathbb{P}$-a.s. over the set $\left\{\tau_{n}<\infty\right\}$ and

$$
\lim _{n \rightarrow \infty} \tau_{n}=\infty \quad \mathbb{P} \text {-a.s.; }
$$

(ii) $\left\{i_{n}\right\}_{n \geq 1}$ is a sequence of $\mathbb{R}_{++}$-valued random variables such that $i_{n}$ is $\mathscr{F}_{\tau_{n}}$-measurable for every $n \geq 1$;

(iii) The following integrability condition holds:

$$
\sum_{n \geq 1} \mathbb{E}\left[e^{-\rho \tau_{n}}\left(i_{n}+1\right)\right]<\infty
$$

For $n \geq 1, \tau_{n}$ represents an intervention time, whereas $i_{n}$ represents the intervention size at the corresponding intervention time $\tau_{n}$. Condition (2.3) ensures that, within a finite time interval, only a finite number of actions are executed. We allow the case $\tau_{n}=\infty$ definitively, meaning that only a finite number of actions are taken. Condition (2.4) ensures that the functional defined below is well defined. We call null control any sequence $\left\{\left(\tau_{n}, i_{n}\right)\right\}_{n \geq 1}$ such that $\tau_{n}=\infty$ for each $n \geq 1$ and denote any of them by $\varnothing$. Notice that using the same notation $\varnothing$ for the null controls is not ambiguous with regard to the control problem we are going to define, as any null control will give rise to the same payoff.

Given a control $I \in \mathscr{I}$, an initial stopping time $\tau \geq 0$ and a random variable $\xi>0 \mathbb{P}$-a.s. $\mathscr{F}_{\tau^{-}}$ measurable, we denote by $X^{\tau, \xi, I}=\left\{X_{r}^{\tau, \xi, I}\right\}_{r \in[0, \infty)}$ the unique (up to indistinguishability) càdlàg process on $[\tau, \infty$ ) solving the SDE (in integral form)

$$
X_{t}^{\tau, \xi, I}= \begin{cases}0 & \text { for } t \in[0, \tau) \\ \xi+\int_{\tau}^{t} b\left(X_{s}^{\tau, \xi, I}\right) d s+\int_{\tau}^{t} \sigma\left(X_{s}^{\tau, \xi, I}\right) d W_{s}+\sum_{n \geq 1} \mathbf{1}_{[\tau, r]}\left(\tau_{n}\right) \cdot i_{n} & \text { for } t \in[\tau, \infty)\end{cases}
$$

If $t=0$ and $\xi \equiv x \in \mathbb{R}_{++}$then we denote $X^{0, \xi, I}$ by $X^{x, I}$. It is easily seen that, if $\tau^{\prime}$ is another stopping time such that $\tau^{\prime} \geq \tau$, then the following flow property holds true

$$
X_{t}^{\tau, \xi, I}=X_{t}^{\tau^{\prime}, X_{\tau^{\prime-}, \xi}^{\tau, \xi, I}} \forall t \geq \tau^{\prime}, \mathbb{P} \text {-a.e.. }
$$

Note that, up to undistinguishability, we have $X^{x, \varnothing}=Z^{0, x}$. Moreover, setting by convention $\tau_{0}:=0$, $i_{0}:=0$, and $X_{0^{-}}:=x$, we have recursively on $n \in \mathbb{N}$

$$
X_{t}^{x, I}=Z_{t}^{\tau_{n}, X_{\tau_{n}}^{x, I}} \forall t \in\left[\tau_{n}, \tau_{n+1}\right), \mathbb{P} \text {-a.s.. }
$$

Then, by (2.2), we have the following monotonicity of the controlled process with respect to the initial data

$$
X_{t}^{x, I} \leq X_{t}^{x^{\prime}, I} \mathbb{P} \text {-a.s., } \forall t \geq 0, \forall I \in \mathscr{I}, \forall x, x^{\prime}: 0<x \leq x^{\prime} .
$$

Next, we introduce the optimization problem. Given $\rho>0, f: \mathbb{R}_{++} \rightarrow \mathbb{R}_{++}$measurable, $c_{0}>0$, $c_{1}>0$, we define the payoff functional $J$ by

$$
J(x, I):=\mathbb{E}\left[\int_{0}^{\infty} e^{-\rho t} f\left(X_{t}^{x, I}\right) d t-\sum_{n \geq 1} e^{-\rho \tau_{n}}\left(c_{0} i_{n}+c_{1}\right)\right], \quad \forall x \in \mathbb{R}_{+}, \forall I \in \mathscr{I} .
$$

We notice that (2.4) and the fact that $f$ is bounded from below ensure that $J(x, I)$ is well defined and takes values in $\mathbb{R} \cup\{\infty\}$.

We will make use of the following assumption on $f$. 
Assumption 2.3. $f \in C^{1}\left(\mathbb{R}_{++} ; \mathbb{R}_{+}\right), f^{\prime}>0, f^{\prime}$ is strictly decreasing, and $f$ satisfies the Inada condition at $\infty$ :

$$
f^{\prime}(\infty):=\lim _{x \rightarrow \infty} f^{\prime}(x)=0 .
$$

Finally, without loss of generality, we assume that $f\left(0^{+}\right):=\lim _{x \rightarrow 0^{+}} f(x)=0$.

Note that

$$
M_{b}:=\left(\sup _{x \in \mathbb{R}_{++}} b^{\prime}(x)\right)^{+}<\infty
$$

by Assumption 2.1. The following assumption will ensure finiteness for the problem (Proposition 3.2).

Assumption 2.4. $\rho>M_{b}$.

Assumptions 2.1, 2.3, and 2.4 will be standing through the rest of the manuscript.

The optimal control problem that we address consists in maximizing the functional (2.8) over $I \in \mathscr{I}$, i.e., for each $x \in \mathbb{R}_{+}$, we consider the maximization problem

$$
\sup _{I \in \mathscr{I}} J(x, I)
$$

Remark 2.5. The fact that $c_{1}>0$ means that there is a fixed cost when the investment occurs. This provides that $(\mathbf{P})$ is well posed as an impulse control problem, i.e. optimal controls can be found within the class of impulse controls. If it was $c_{1}=0$ (only proportional intervention cost), the setting providing existence of optimal controls would be the more general singular control setting (see e.g. [63, Ch. 4]). For comparison between impulse and singular control we refer to [18]; for the relevance of the introduction of the fixed cost we refer to [61], where the asymptotics for $c_{1} \rightarrow 0$ is investigated. In Subsection 7.1.2, we comment this issue through the numerical outputs.

We also notice that one might consider more general intervention costs $C: \mathbb{R}_{++} \rightarrow \mathbb{R}_{+}$increasing and convex, (e.g. $C(i)=\alpha i^{2}+\beta i+c_{1}$ with $\alpha, c_{1}>0$ and $\beta \geq 0$ ). We believe that, at least for a suitable subclass of such cost functons, the solution would depict the same structure as the one we provide here in the affine case (i.e. $C(i)=c_{0} i+c_{1}$ ). On the other hand, we underline that at many points our proofs make use of the affine structure of the cost and the generalization seems to be not straightforward.

\section{Preliminary results on the value function}

In this section we introduce the value function associated with $(\mathbf{P})$ and establish some basic properties of it. We define the value function $v$ by

$$
v(x):=\sup _{I \in \mathscr{I}} J(x, I), \quad \forall x \in \mathbb{R}_{++} .
$$

We notice that $v$ is $\overline{\mathbb{R}}_{+}$-valued, as by Assumption 2.3

$$
v(x) \geq J(x, \varnothing)=\hat{v}(x):=\mathbb{E}\left[\int_{0}^{\infty} e^{-\rho t} f\left(X_{t}^{x, \varnothing}\right) d t\right] \geq 0 \quad \forall x \in \mathbb{R}_{++} .
$$

Note that $\hat{v}$ is nondecreasing as $f^{\prime}>0$ (Assumption 2.3) and by (2.7). 
Proposition 3.1. $v$ is nondecreasing.

Proof. Let $0<x \leq x^{\prime}$. Since $f^{\prime}>0$ (see Assumption 2.3), from (2.7) we get $J(x ; I) \leq J\left(x^{\prime} ; I\right)$ for every $I \in \mathscr{I}$. The claim follows by taking the supremum over $I \in \mathscr{I}$.

We denote by $f^{*}$ the Fenchel-Legendre transform of $f$ on $\mathbb{R}_{++}$:

$$
f^{*}(\alpha):=\sup _{x \in \mathbb{R}_{++}}\{f(x)-\alpha x\}, \quad \forall \alpha \in \mathbb{R}_{++} .
$$

Nonnegativity and continuity of $f$ (see Assumption 2.3) and the condition $f^{\prime}(\infty)=0$ (again Assumption 2.3) guarantee that $0 \leq f^{*}(\alpha)<\infty$ for all $x \in \mathbb{R}_{++}$.

Proposition 3.2. For all $\alpha \in\left(0, c_{0} \rho\right]$ we have

$$
0 \leq \hat{v}(x) \leq v(x) \leq \frac{f^{*}(\alpha)}{\rho}+\frac{\alpha x}{\rho}, \quad \forall x \in \mathbb{R}_{++}
$$

and

$$
\limsup _{x \rightarrow \infty} \frac{v(x)}{x}=0 .
$$

Proof. The fact that $0 \leq \hat{v} \leq v$ was already noticed in (3.2). We show the remaining inequality. Let $x \in \mathbb{R}_{++}$and $I \in \mathscr{I}$. For $R>0$, define the stopping time $\hat{\tau}_{R}:=\inf \left\{t \geq 0: X_{t}^{x, I} \geq R\right\}$. Notice that, since $b \in C^{1}\left(\mathbb{R}_{++} ; \mathbb{R}\right)$ and $b(0)=0$ by Assumption 2.1, mean value theorem yields

$$
b(\xi) \leq b(0)+M_{b} \xi=M_{b} \xi, \quad \forall \xi \in \mathbb{R},
$$

where $M_{b}$ is defined in (2.9). Set $\tau_{0}:=0$ and let $t \in \mathbb{R}_{+_{+}}$. Applying Itô's formula to $\varphi\left(s, X_{s}^{x, I}\right):=$ $e^{-\rho s} X_{s}^{x, I}, s \in\left[0, \hat{\tau}_{R}\right)$, taking expectations after considering that $X_{s}^{x, I} \in(0, R)$ for $s \in\left[0, \hat{\tau}_{R}\right)$, summing up over $n \in \mathbb{N}$, and using (3.6) and 2.4, we get

$$
\begin{aligned}
\mathbb{E}\left[e^{-\rho t} X_{t \wedge \hat{\tau}_{R}}^{x, I}\right] & =x-\rho \int_{0}^{t} e^{-\rho s} \mathbb{E}\left[\mathbf{1}_{\left[0, \hat{t}_{R}\right]}(s) X_{s}^{x, I}\right] d s+\int_{0}^{t} e^{-\rho s} \mathbb{E}\left[\mathbf{1}_{\left[0, \hat{t}_{R}\right]}(s) b\left(X_{s}^{x, I}\right)\right] d s+e^{-\rho t} \mathbb{E}\left[\sum_{n \geq 1, \tau_{n} \leq t \wedge \hat{\tau}_{R}} i_{n}\right] \\
& \leq x+\left(M_{b}-\rho\right) \int_{0}^{t} e^{-\rho s} \mathbb{E}\left[\mathbf{1}_{\left[0, \hat{t}_{R}\right]}(s) X_{s}^{x, I}\right] d s+e^{-\rho t}\left[\sum_{n \geq 1, \tau_{n} \leq t \wedge \hat{\tau}_{R}} i_{n}\right] \\
& \leq x+e^{-\rho t} \mathbb{E}\left[\sum_{n \geq 1, \tau_{n} \leq t \wedge \hat{\tau}_{R}} i_{n}\right] .
\end{aligned}
$$

By Fatou's lemma, letting $R \rightarrow \infty$ and observing that $\tau_{R} \rightarrow \infty \mathbb{P}$-a.s. , we get

$$
\mathbb{E}\left[e^{-\rho t} X_{t}^{x, I}\right] \leq x+e^{-\rho t} \mathbb{E}\left[\sum_{n \geq 1, \tau_{n} \leq t} i_{n}\right] .
$$

By integrating the second term on the right-hand side of (3.7), we have using Fubini-Tonelli's Theorem (as all the integrands involved are nonnegative)

$$
\mathbb{E}\left[\int_{0}^{\infty}\left(e^{-\rho t} \sum_{n \geq 1, \tau_{n} \leq t} i_{n}\right) d t\right]=\mathbb{E}\left[\sum_{n \geq 1}\left(\int_{\tau_{n}}^{\infty} e^{-\rho\left(t-\tau_{n}\right)} d t\right) e^{-\rho \tau_{n}} i_{n}\right]=\frac{1}{\rho} \mathbb{E}\left[\sum_{n \geq 1} e^{-\rho \tau_{n}} i_{n}\right] .
$$


Therefore, taking into account (3.7), (3.8) and (2.4), we have

$$
\mathbb{E}\left[\int_{0}^{\infty} e^{-\rho t} X_{t}^{x, I} d t\right] \leq \frac{1}{\rho}\left(x+\mathbb{E}\left[\sum_{n \geq 1} e^{-\rho \tau_{n}} i_{n}\right]\right)<\infty .
$$

Now let $\alpha>0$. By definition of $f^{*}$ and by (3.9), we can write

$$
\begin{aligned}
\mathbb{E}\left[\int_{0}^{\infty} e^{-\rho t} f\left(X_{t}^{x, I}\right) d t-\sum_{n \geq 1} e^{-\rho \tau_{n}}\left(c_{0} i_{n}+c_{1}\right)\right] & \leq \mathbb{E}\left[\int_{0}^{\infty} e^{-\rho t}\left(f^{*}(\alpha)+\alpha X_{t}^{x, I}\right) d t-\sum_{n \geq 1} e^{-\rho \tau_{n}}\left(c_{0} i_{n}+c_{1}\right)\right] \\
& \leq \frac{f^{*}(\alpha)}{\rho}+\frac{\alpha x}{\rho}+\left(\frac{\alpha}{\rho}-c_{0}\right) \mathbb{E}\left[\sum_{n \geq 1} e^{-\rho \tau_{n}} i_{n}\right] .
\end{aligned}
$$

By arbitrariness of $I \in \mathscr{I}$, if $\alpha \in\left(0, c_{0} \rho\right]$, the latter provides the last inequality in (3.4).

Take now $\alpha \in\left(0, c_{0} \rho\right]$. By (3.4) we have

$$
0 \leq \limsup _{x \rightarrow \infty} \frac{v(x)}{x} \leq \alpha \limsup _{x \rightarrow \infty} \frac{v(x)}{\alpha x} \leq \alpha \limsup _{x \rightarrow \infty}\left\{\frac{f^{*}(\alpha)}{\alpha \rho x}+\frac{1}{\rho}\right\}=\frac{\alpha}{\rho}
$$

By arbitrariness of $\alpha$ we get (3.5).

Assumption 3.3. The following conditions hold true.

(i) $\rho>\max \left\{B_{0}, C_{0}\right\}$ where $B_{0}, C_{0}$ are the constants defined in Lemma A.3.

(ii) For each $\beta>0$,

$$
M(\beta):=\mathbb{E}\left[\int_{0}^{\infty} e^{-\rho t}\left(f^{\prime}\left(X_{t}^{\beta, \varnothing}\right)\right)^{2} d t\right]<\infty
$$

(iii) For each $\eta>0$, the function $f$ is semiconvex on $[\eta, \infty)$. Precisely, there exists a nonincreasing function $K_{0}: \mathbb{R}_{++} \rightarrow \mathbb{R}_{++}$such that

$$
f(\lambda x+(1-\lambda) y)-\lambda f(x)-(1-\lambda) f(y) \leq K_{0}(\eta) \lambda(1-\lambda)(y-x)^{2}, \quad \forall \lambda \in[0,1], \forall x, y \in[\beta, \infty) .
$$

(iv) The function $K_{0}$ in (iii) is such that, for each $\beta>0$,

$$
\hat{M}(\beta):=\mathbb{E}\left[\int_{0}^{\infty} e^{-\rho t}\left(K_{0}\left(X_{t}^{\beta, \varnothing}\right)\right)^{2} d t\right]<\infty .
$$

Remark 3.4. Semiconvex functions are functions that can be written as difference of a convex function and a quadratic one (see [26, Prop. 1.1.3] or [72, Ch. 4, Sec. 4.2]). Moreover, a function $\varphi \in C^{2}([\beta, \infty) ; \mathbb{R})$ verifies (3.11) with $K_{0}(\eta):=-2 \inf _{[\eta, \infty)} f^{\prime \prime}$ (see again [26, Prop. 1.1.3]).

The following Proposition shows that power functions satisfy Assumption 3.3(ii)-(iv).

Proposition 3.5. Let $f \in C^{2}\left(\mathbb{R}_{++} ; \mathbb{R}\right)$ such that $f^{\prime}>0$, $f^{\prime \prime}<0$, and

$$
f^{\prime}(\xi) \leq C_{0}\left(1+|\xi|^{\gamma-1}\right), \quad f^{\prime \prime}(\xi) \geq-C_{0}\left(1+|\xi|^{\gamma-2}\right) \quad \forall \xi \in \mathbb{R}_{++}
$$

for some $C_{0}>0$ and $\gamma \in(0,1)$, and let $\rho>L_{b}(1-\gamma)+\frac{1}{2} L_{\sigma}^{2}(1-\gamma)(2-\gamma)$. Then $f$ satisfies Assumptions 3.3(ii)-(iv). 
Proof. Let $\beta \in \mathbb{R}_{++}$and observe that, by Assumption 2.1, we have

$$
|b(\xi)| \leq L_{b}|\xi|, \quad|\sigma(\xi)| \leq L_{\sigma}|\xi| \quad \forall \xi \in \mathbb{R} .
$$

. With a localization procedure similar to the one of the prof of Proposition 3.2 (now keeping the process $X^{\beta, \varnothing}$ away from 0), we get from Itô's formula

$$
\begin{aligned}
& \mathbb{E}\left[e^{-\rho t}\left|X_{t}^{\beta, \varnothing}\right|^{\gamma-1}\right] \\
& =|\beta|^{\gamma-1}+\mathbb{E}\left[\int_{0}^{t} e^{-\rho s}\left[-\rho\left|X_{s}^{\beta, \varnothing}\right|^{\gamma-1}+(\gamma-1)\left|X_{s}^{\beta, \varnothing}\right|^{\gamma-2} b\left(X_{s}^{\beta, \varnothing}\right)+\frac{1}{2}(\gamma-1)(\gamma-2)\left|X_{s}^{\beta, \varnothing}\right|^{\gamma-3} \sigma^{2}\left(X_{s}^{\beta, \varnothing}\right)\right] d s\right] \\
& \leq|\beta|^{\gamma-1}+\mathbb{E}\left[\int_{0}^{t} e^{-\rho s}\left[-\rho\left|X_{s}^{\beta, \varnothing}\right|^{\gamma-1}+L_{b}(1-\gamma)\left|X_{s}^{\beta, \varnothing}\right|^{\gamma-1}+\frac{1}{2} L_{\sigma}^{2}(1-\gamma)(2-\gamma)\left|X_{s}^{\beta, \varnothing}\right|^{\gamma-1}\right] d s\right] .
\end{aligned}
$$

Then Assumption 3.3(ii) follows from (3.13) and Gronwall's Lemma applied to the inequality above.

Moreover, note that, since $\xi \mapsto-C_{0}\left(1+|\xi|^{\gamma-2}\right)$ is negative and increasing, by Remark 3.4 and (3.13) we obtain that $f$ verifies Assumption 3.3(iii) with

$$
K_{0}(\eta):=-2 \gamma(\gamma-1) \eta^{\gamma-2} \quad \forall x \in \mathbb{R}_{++}
$$

Finally, similarly as above, we have

$$
\begin{aligned}
& \mathbb{E}\left[e^{-\rho t}\left|X_{t}^{\beta, \varnothing}\right|^{\gamma-2}\right] \\
& =|\beta|^{\gamma-2}+\mathbb{E}\left[\int_{0}^{t} e^{-\rho s}\left[-\rho\left|X_{s}^{\beta, \varnothing}\right|^{\gamma-2}+(\gamma-2)\left|X_{s}^{\beta, \varnothing}\right|^{\gamma-3} b\left(X_{s}^{\beta, \varnothing}\right)+\frac{1}{2}(\gamma-2)(\gamma-3)\left|X_{s}^{\beta, \varnothing}\right|^{\gamma-4} \sigma^{2}\left(X_{s}^{\beta, \varnothing}\right)\right] d s\right] \\
& \leq|\beta|^{\gamma-2}+\mathbb{E}\left[\int_{0}^{t} e^{-\rho s}\left[-\rho\left|X_{s}^{\beta, \varnothing}\right|^{\gamma-2}+L_{b}(1-\gamma)\left|X_{s}^{\beta, \varnothing}\right|^{\gamma-1}+\frac{1}{2} L_{\sigma}^{2}(1-\gamma)(2-\gamma)\left|X_{s}^{\beta, \varnothing}\right|^{\gamma-1}\right] d s\right] .
\end{aligned}
$$

Then Assumption 3.3(iv) follows from Gronwall's Lemma applied to the inequality above and from (3.14).

Remark 3.6. Note that, if $\rho$ satisfies Assumption 3.3(i), then it also satisfies the requirement of Proposition 3.5.

Proposition 3.7. Let Assumption 3.3 hold. Then $v$ is semiconvex on $[\beta, \infty)$ for each $\beta>0$, i.e., for each $\beta>0$ there exists $K_{1}(\beta)>0$ such that

$$
v(\lambda x+(1-\lambda) y)-\lambda v(x)-(1-\lambda) v(y) \leq K_{1}(\beta) \lambda(1-\lambda)(x-y)^{2} \quad \forall \lambda \in[0,1], \forall x, y \in[\beta, \infty) .
$$

Proof. Fix $\beta>0$. Let $x, y \in[\beta, \infty)$ with $x \leq y$, and $I \in \mathscr{I}$. For each $\lambda \in[0,1]$ set $z_{\lambda}:=\lambda x+(1-\lambda) y$ and $\Sigma^{\lambda, x, y, I}:=\lambda X^{x, I}+(1-\lambda) X^{y, I}$. We write

$$
\begin{aligned}
& J\left(z_{\lambda}, I\right)-\lambda J(x, I)-(1-\lambda) J(y, I)=\mathbb{E}\left[\int_{0}^{\infty} e^{-\rho t}\left(f\left(X_{t}^{z_{\lambda}, I}\right)-\lambda f\left(X_{t}^{x, I}\right)-(1-\lambda) f\left(X_{t}^{y, I}\right)\right) d t\right]= \\
& =\mathbb{E}\left[\int_{0}^{\infty} e^{-\rho t}\left(f\left(X_{t}^{z_{\lambda}, I}\right)-f\left(\Sigma_{t}^{\lambda, x, y, I}\right)\right) d t\right]+\mathbb{E}\left[\int_{0}^{\infty} e^{-\rho t}\left(f\left(\Sigma_{t}^{\lambda, x, y, I}\right)-\lambda f\left(X_{t}^{x, I}\right)-(1-\lambda) f\left(X_{t}^{y, I}\right)\right) d t\right] .
\end{aligned}
$$


Applying Hölder's inequality, observing that $X^{\beta, \varnothing} \leq X^{z_{\lambda}, I} \wedge \Sigma^{\lambda, x, y, I}$, that $f^{\prime}$ is decreasing, using Assumption 3.3(i), and using Lemma A.3(ii), we write

$$
\begin{aligned}
\mathbf{A} & \leq \mathbb{E}\left[\int_{0}^{\infty} e^{-\rho t} f^{\prime}\left(X_{t}^{\beta, \varnothing}\right)\left|X_{t}^{z_{\lambda}, I}-\Sigma_{t}^{\lambda, x, y, I}\right| d t\right] \\
& \leq\left(\mathbb{E}\left[\int_{0}^{\infty} e^{-\rho t}\left(f^{\prime}\left(X_{t}^{\beta, \varnothing}\right)\right)^{2} d t\right]\right)^{1 / 2}\left(\mathbb{E}\left[\int_{0}^{\infty} e^{-\rho t}\left|X_{t}^{z_{\lambda}, I}-\Sigma_{t}^{\lambda, x, y, I}\right|^{2} d t\right]\right)^{1 / 2} \\
& \leq M(\beta)^{1 / 2}\left(\mathbb{E}\left[\int_{0}^{\infty} e^{-\rho t}\left|X_{t}^{z_{\lambda}, I}-\Sigma_{t}^{\lambda, x, y, I}\right|^{2} d t\right]\right)^{1 / 2} \\
& \leq M(\beta)^{1 / 2}\left(\int_{0}^{\infty} e^{-\rho t} A_{0} e^{B_{0} t} d t\right)^{1 / 2} \lambda(1-\lambda)|x-y|^{2} \\
& =\frac{A_{0}^{1 / 2} M(\beta)}{\left(\rho-B_{0}\right)^{1 / 2}} \lambda(1-\lambda)|x-y|^{2} .
\end{aligned}
$$

Moreover, by Assumption 3.3(i),(iii),(iv), again using Hölder's inequality and applying Lemma A.3(i), we have

$$
\begin{aligned}
\mathbf{B} & \leq \lambda(1-\lambda) \mathbb{E}\left[\int_{0}^{\infty} e^{-\rho t} K_{0}\left(X_{t}^{\beta, \varnothing}\right)\left|X_{t}^{y, I}-X_{t}^{x, I}\right|^{2} d t\right] \\
& \leq \lambda(1-\lambda)\left(\mathbb{E}\left[\int_{0}^{\infty} e^{-\rho t}\left(K_{0}\left(X_{t}^{\beta, \varnothing}\right)\right)^{2} d t\right]\right)^{1 / 2}\left(\mathbb{E}\left[\int_{0}^{\infty} e^{-\rho t}\left|X_{t}^{y, I}-X_{t}^{x, I}\right|^{4} d t\right]\right)^{1 / 2} \\
& \leq \lambda(1-\lambda) \hat{M}(\beta)^{1 / 2}\left(\int_{0}^{\infty} e^{-\rho t} e^{C_{0} t} d t\right)^{1 / 2}|x-y|^{2} \\
& =\frac{\hat{M}(\beta)^{1 / 2}}{\left(\rho-C_{0}\right)^{1 / 2}} \lambda(1-\lambda)|x-y|^{2} .
\end{aligned}
$$

Now let $\delta>0$ and let $I$ be such that $v(z) \leq J(z, I)+\delta$. The inequalities above provide

$$
\begin{aligned}
v(z)-\delta-\lambda v(x)-(1-\lambda) v(y) & \leq J(z, I)-\lambda J(x, I)-(1-\lambda) J(y, I) \\
& \leq K_{1}(\beta) \lambda(1-\lambda)|x-y|^{2} \quad \forall x, y \geq \beta, \forall \lambda \in[0,1],
\end{aligned}
$$

where $K_{1}(\beta):=\frac{\hat{M}(\beta)}{\left(\rho-C_{0}\right)^{1 / 2}}+\frac{A_{0}^{1 / 2} \hat{M}(\beta)}{\left(\rho-B_{0}\right)^{1 / 2}}$. We then obtain (3.15) by arbitrariness of $\delta$.

In view of the fact that the results which follow rely on the semiconvexity of $v$, Assumption 3.3 will be standing for the remaining of this section and in Sections 4, 5, 6 .

Define the space

$$
\operatorname{Lip}_{\text {loc }, c_{0}}\left(\mathbb{R}_{++}\right):=\left\{u: \mathbb{R}_{++} \rightarrow \mathbb{R} \text { locally Lipschitz continuous on } \mathbb{R}_{++} \text {, s.t. } \limsup _{x \rightarrow \infty} \frac{u(x)}{x}<c_{0}\right\} \text {. }
$$

We recall that semiconvex functions on open sets are locally Lipschitz. So, by Propositions 3.2 and 3.7, we have $v \in \operatorname{Lip}_{\text {loc, } c_{0}}\left(\mathbb{R}_{++}\right)$. The space Lip loc, $c_{0}\left(\mathbb{R}_{++}\right)$will be used in the next section.

\section{Dynamic Programming}

The dynamic programming equation associated to our dynamic optimization problem is the quasivariational inequality (see, e.g., [17])

$$
\min \{\mathscr{L} u-f, u-\mathscr{M} u\}=0,
$$


where $\mathscr{L}$ and $\mathscr{M}$ are operators formally defined by

$$
\begin{gathered}
\mathscr{L} u(x):=\rho u(x)-b(x) u^{\prime}(x)-\frac{1}{2} \sigma^{2}(x) u^{\prime \prime}(x), \quad x \in \mathbb{R}_{++}, \\
\mathscr{M} u(x):=\sup _{i>0}\left\{u(x+i)-c_{0} i-c_{1}\right\}, \quad x \in \mathbb{R}_{++} .
\end{gathered}
$$

We note that $\mathscr{L}$ is a differential operator, so it has a local nature, while $\mathscr{M}$ is a functional operator having a nonlocal nature.

\subsection{Continuation and action region}

Here we define and study the first properties of the continuation and action region in the state space $\mathbb{R}_{++}$.

Lemma 4.1. $\mathscr{M}$ maps $\operatorname{Lip}_{l o c, c_{0}}\left(\mathbb{R}_{++}\right)$into itself.

Proof. Let $u \in \operatorname{Lip}_{\text {loc, } c_{0}}\left(\mathbb{R}_{++}\right)$. Then there exists $\bar{x}, \varepsilon>0$ such that

$$
\frac{u(x)}{x}-c_{0} \leq-\varepsilon \quad \forall x \geq \bar{x} .
$$

By (4.3), for all $i>0, x \geq \bar{x}$, we have

$$
u(x+i)-\left(c_{0} i+c_{1}\right)=(x+i)\left(\frac{u(x+i)}{x+i}-c_{0}\right)+c_{0} x-c_{1} \leq\left(c_{0}-\varepsilon\right) x .
$$

Hence, by taking the supremum over $i>0$,

$$
\frac{\mathscr{M} u(x)}{x} \leq c_{0}-\varepsilon \quad \forall x \geq \bar{x}
$$

which shows that $\limsup _{x \rightarrow \infty} \frac{\mathscr{M} u(x)}{x}<c_{0}$.

Now we show that $\mathscr{M} u$ is Lipschitz continuous on $\left[M^{-1}, M\right]$ for each $M>0$. Using (4.3) one can show that

$$
\limsup _{i \rightarrow+\infty} \sup _{x \in\left[M^{-1}, M\right]}\left\{u(x+i)-c_{0} i\right\}=-\infty
$$

Set

$$
U(x):=\sup \left\{i \in \mathbb{R}_{++}: u(x+i)-c_{0} i \geq u(x)-1\right\} \quad \forall x \in\left[M^{-1}, M\right] .
$$

The limit (4.4) provides that there exists $R>0$ such that

$$
U(x) \leq R \quad \forall x \in\left[M^{-1}, M\right] .
$$

Hence, we have

$$
\mathscr{M} u(x)=\sup _{i \in(0, R]}\left\{u(x+i)-c_{0} i-c_{1}\right\} \quad \forall x \in\left[M^{-1}, M\right] .
$$

Now let $\hat{L}$ be the Lipschitz constant of $\left.u\right|_{\left[M^{-1}, M+R\right]}$. Then, if $M^{-1} \leq x<y \leq M, 0<i \leq R$, we can write

$$
u(x+i)-\left(c_{0} i+c_{1}\right)-\hat{L}(y-x) \leq u(y+i)-\left(c_{0} i+c_{1}\right) \leq u(x+i)-\left(c_{0} i+c_{1}\right)+\hat{L}(y-x) .
$$

Now the claim follows by taking the supremum over $i \in(0, R]$ on (4.6) and recalling (4.5). 
By definition of $v$ we have

$$
v(x) \geq v(x+i)-c_{0} i-c_{1} \quad \forall i>0
$$

hence

$$
v \geq \mathscr{M} v .
$$

We define the continuation region $\mathscr{C}$ and the action region $\mathscr{A}$ by

$$
\begin{array}{ll}
\mathscr{C}:=\left\{x \in \mathbb{R}_{++}: \mathscr{M} v(x)<v(x)\right\} & \text { (continuation region) } \\
\mathscr{A}:=\mathbb{R}_{++} \backslash \mathscr{C}=\left\{x \in \mathbb{R}_{++}: \mathscr{M} v(x)=v(x)\right\} & \text { (action region). }
\end{array}
$$

They will represent, respectively, the region where it will be convenient to let the system evolve autonomously and the region where it wil be convenient to undertake an action by exercising an impulse. By Proposition 3.2 and Lemma 4.1, both members of (4.8) are finite continuous functions. In particular, $\mathscr{C}$ is open and $\mathscr{A}$ is closed in $\mathbb{R}_{++}$.

For $x \in \mathscr{A}$, let us introduce the set

$$
\Xi(x):=\underset{i>0}{\operatorname{argmax}}\left\{v(x+i)-c_{0} i-c_{1}\right\} .
$$

Clearly $\Xi(x)$ is empty if $x \in \mathscr{C}$. In principle $\Xi(x)$ might be empty even if $x \in \mathscr{A}$, but this is not the case as shown by the following.

Proposition 4.2. Let $x \in \mathscr{A}$.

(i) $\Xi(x)$ is not empty.

(ii) For all $\xi \in \Xi(x)$, we have $x+\xi \in \mathscr{C}$.

Proof. (i) Let $x \in \mathscr{A}$ and take a sequence $\left\{i_{n}\right\}_{n \in \mathbb{N} \backslash\{0\}} \subset \mathbb{R}_{++}$such that

$$
\mathscr{M} v(x) \geq v\left(x+i_{n}\right)-c_{0} i_{n}-c_{1} \geq \mathscr{M} v(x)-\frac{1}{n}, \quad \forall n \in \mathbb{N} \backslash\{0\} .
$$

Then, considering that $\limsup _{i \rightarrow \infty} \frac{v(x+i)}{x+i}=0$ by Proposition 3.2 and that $\mathscr{M} v(x)$ is finite, we easily see, arguing by contradiction, that, in order to fulfill (4.11), the sequence $\left\{i_{n}\right\}_{n \in \mathbb{N}}$ must be bounded. Hence, by considering a subsequence if necessary, we have $i_{n} \rightarrow i^{*} \in \mathbb{R}_{+}$. Let us show that $i^{*}>0$. Indeed, assume by contradiction that $i^{*}=0$. By (4.11), taking into account that $v$ is continuous and that $v(x)=\mathscr{M} v(x)$ as $x \in \mathscr{A}$, we obtain $v(x)=\mathscr{M} v(x) \leq v(x)-c_{1}$, a contradiction. Then we have shown that $i^{*}>0$. From (4.11) we obtain, by continuity, $\mathscr{M} v(x)=v\left(x+i^{*}\right)-c_{0} i^{*}-c_{1}$ and the claim follows.

(ii) This part of the proof closely follows the proof of [43, Prop. 2]. We omit it for brevity.

Note that, as a consequence of Proposition 4.2 , we have $\mathscr{C} \neq \varnothing$. Indeed, either $\mathscr{A}=\varnothing$, thus $\mathscr{C}=\mathbb{R}_{++} ;$or $\mathscr{A} \neq \varnothing$, thus $\mathscr{C} \neq \varnothing$ by Proposition 4.2(ii). Formally, Proposition 4.2(ii) says that, if the system is in a position $x \in \mathscr{A}$ : (i) an optimal control exists (part (i)); (ii) this optimal control places the system in $\mathscr{C}$ (part (ii)). We will verify this fact rigorously afterwards. 


\subsection{Dynamic Programming Principle and viscosity solutions}

The rigorous connection between $v$ and (QVI) passes through the dynamic programming principle (DPP).

Proposition 4.3. For every $x>0$ and every $\mathbb{F}$-stopping time $\tau \in \overline{\mathbb{R}}_{+}$,

$$
v(x)=\sup _{I \in \mathscr{I}} \mathbb{E}\left[\int_{0}^{\tau} e^{-\rho s} f\left(X_{s}^{x, I}\right) d s-\sum_{n \geq 1, \tau_{n} \leq \tau} e^{-\rho \tau_{n}}\left(c_{0} i_{n}+c_{1}\right)+e^{-\rho \tau} v\left(X_{\tau}^{x, I}\right)\right] .
$$

Proof. We refer to [22] (for the finite horizon case; our formulation is the usual one for time homogeneous infinite horizon problems).

Here we study (QVI) by means of viscosity solutions.

Definition 4.4 (Viscosity Solution). Let $u \in \operatorname{Lip}_{l o c, c_{0}}\left(\mathbb{R}_{++}\right)$.

(i) $u$ is a viscosity subsolution to $(\mathbf{Q V I})$ if for every $\left(x_{0}, \varphi\right) \in \mathbb{R}_{++} \times C^{2}\left(\mathbb{R}_{++}\right)$such that $u-\varphi$ has a local maximum at $x_{0}$ and $u\left(x_{0}\right)=\varphi\left(x_{0}\right)$ we have

$$
\min \left\{\mathscr{L} \varphi\left(x_{0}\right)-f\left(x_{0}\right), u\left(x_{0}\right)-\mathscr{M} u\left(x_{0}\right)\right\} \leq 0 ;
$$

(ii) $u$ is a viscosity supersolution to $(\mathbf{Q V I})$ if for every $\left(x_{0}, \varphi\right) \in \mathbb{R}_{++} \times C^{2}\left(\mathbb{R}_{++}\right)$such that $u-\varphi$ has a local minimum at $x_{0}$ and $u\left(x_{0}\right)=\varphi\left(x_{0}\right)$ we have

$$
\min \left\{\mathscr{L} \varphi\left(x_{0}\right)-f\left(x_{0}\right), u\left(x_{0}\right)-\mathscr{M} u\left(x_{0}\right)\right\} \geq 0
$$

(iii) $u$ is a viscosity solution to (QVI) if it is both a viscosity subsolution and a viscosity supersolution of (QVI).

Proposition 4.5. The value function $v$ is a viscosity solution of (QVI).

Proof. Supersolution property. Let $x_{0} \in \mathbb{R}_{++}$and $\varphi \in C^{2}\left(\mathbb{R}_{++}\right)$be such that $v-\varphi$ has a local minimum at $x_{0}$ and $v\left(x_{0}\right)=\varphi\left(x_{0}\right)$. In particular, $v \geq \varphi$ on $\left(x_{0}-\delta, x_{0}+\delta\right)$ for a suitable $\delta \in\left(0, x_{0}\right)$. By (4.8) we only need to show that $\mathscr{L} \varphi\left(x_{0}\right)-f\left(x_{0}\right) \geq 0$. To this aim, consider the stopping time $\tau:=\inf \left\{t \geq 0:\left|X_{t}^{x_{0}, \varnothing}-x_{0}\right|>\delta\right\}$, and note that $\mathbb{P}\{\tau>0\}=1$ by continuity of trajectories. Then, from (DPP) we get

$$
v\left(x_{0}\right) \geq \mathbb{E}\left[\int_{0}^{\tau \wedge \varepsilon} e^{-\rho t} f\left(X_{t}^{x_{0}, \varnothing}\right) d t+e^{-\rho(\tau \wedge \varepsilon)} v\left(X_{\tau \wedge \varepsilon}^{x_{0}, \varnothing}\right)\right] \quad \forall \varepsilon>0 .
$$

From this we derive

$$
\varphi\left(x_{0}\right) \geq \mathbb{E}\left[\int_{0}^{\tau \wedge \varepsilon} e^{-\rho t} f\left(X_{t}^{x_{0}, \varnothing}\right) d t+e^{-\rho(\tau \wedge \varepsilon)} \varphi\left(X_{\tau \wedge \varepsilon}^{x_{0}, \varnothing}\right)\right] \quad \forall \varepsilon>0 .
$$

By applying Dynkin's formula, dividing by $\varepsilon$, letting $\varepsilon \rightarrow 0^{+}$, and considering that $X^{x, \varnothing}$ is rightcontinuous in 0 and $\mathbb{P}\{\tau>\varepsilon\} \rightarrow 1$ as $\varepsilon \rightarrow 0^{+}$, we obtain the desired inequality.

Subsolution property. Let $x_{0} \in \mathbb{R}_{++}$and $\varphi \in C^{2}\left(\mathbb{R}_{++}\right)$be such that $v-\varphi$ has a local maximum at $x_{0}$ and $v\left(x_{0}\right)=\varphi\left(x_{0}\right)$. If $v\left(x_{0}\right)=\mathscr{M} v\left(x_{0}\right)$, then we are done. Then assume $v\left(x_{0}\right) \geq \xi+\mathscr{M} v\left(x_{0}\right)$ for some $\xi>0$. In this case, we need to show that $\mathscr{L} \varphi\left(x_{0}\right)-f\left(x_{0}\right) \leq 0$. Assume by contradiction that 
$\mathscr{L} \varphi\left(x_{0}\right)-f\left(x_{0}\right) \geq \varepsilon>0$. By continuity of $\mathscr{L} \varphi-f$ and of $v-\mathscr{M} v$, and in view of the fact that $v-\varphi$ has a local maximum at $x_{0}$ and $\varphi\left(x_{0}\right)=v\left(x_{0}\right)$, there exists $\delta \in\left(0, x_{0} / 2\right)$ such that

$$
\forall x \in B\left(x_{0}, 2 \delta\right] \quad \begin{cases}\text { (i) } & \mathscr{L} \varphi(x)-f(x) \geq \varepsilon / 2 \\ \text { (ii) } & \varphi(x) \geq v(x) \\ \text { (iii) } & v(x)-\mathscr{M} v(x) \geq \xi / 2 .\end{cases}
$$

Now define the stopping time $\tau:=\inf \left\{t \geq 0:\left|X_{t}^{x_{0}, \varnothing}-x_{0}\right|>\delta\right\}$ and note that $\mathbb{P}\{\tau>0\}=1$. In view of (4.14)(iii), undertaking an investment in the region $B\left(x_{0}, 2 \delta\right]$ is not optimal. Hence (DPP) can be rewritten limiting the ranging of $I$ to the set of controls such that $\tau_{1}>\tau$, yielding the simple equality

$$
v\left(x_{0}\right)=\mathbb{E}\left[\int_{0}^{\tau} e^{-\rho t} f\left(X_{t}^{x_{0}, \varnothing}\right) d t+e^{-\rho \tau} v\left(X_{\tau}^{x_{0}, \varnothing}\right)\right] .
$$

Finally, we have, by (4.15), Dynkin's formula, and (4.14)(i)-(ii),

$$
\begin{aligned}
\frac{\varepsilon}{2} \mathbb{E}[\tau] & \leq \mathbb{E}\left[\int_{0}^{\tau} e^{-\rho t}\left(\mathscr{L} \varphi\left(X_{t}^{x_{0}, \varnothing}\right)-f\left(X_{t}^{x_{0}, \varnothing}\right)\right) d t\right] \\
& =\varphi\left(x_{0}\right)-\mathbb{E}\left[\int_{0}^{\tau} e^{-\rho t} f\left(X_{t}^{x_{0}, \varnothing}\right) d t+e^{-\rho \tau} \varphi\left(X_{\tau}^{x_{0}, \varnothing}\right)\right] \\
& \leq v\left(x_{0}\right)-\mathbb{E}\left[\int_{0}^{\tau} e^{-\rho t} f\left(X_{t}^{x_{0}, \varnothing}\right) d t+e^{-\rho \tau} v\left(X_{\tau}^{x_{0}, \varnothing}\right)\right]=0 .
\end{aligned}
$$

This provide a contradiction as $\mathbb{P}\{\tau>0\}=1$.

\subsection{Regularity of the value function}

Here we establish the regularity properties of the value function. Precisely, exploiting the semiconvexity provided by Proposition 3.7 and the viscosity property provided by Proposition 4.5, we show that it is of class $C^{1}$ on $\mathbb{R}_{++}$and of class $C^{2}$ on $\mathscr{C}$.

Theorem 4.6. $v \in C^{1}\left(\mathbb{R}_{++} ; \mathbb{R}\right) \cap C^{2}(\mathscr{C} ; \mathbb{R})$.

Proof. Let $x_{0} \in \mathbb{R}_{++}$. As $v$ is semiconvex in a neighborhood of $x_{0}$ (Proposition 3.7), in such a neighborhood it can be written as difference of a convex function and a quadratic one (see Remark 3.4). Hence, the one-side derivatives $v_{+}^{\prime}\left(x_{0}\right), v_{-}^{\prime}\left(x_{0}\right)$ exist and $v_{-}^{\prime}\left(x_{0}\right) \leq v_{+}^{\prime}\left(x_{0}\right)$. To show that $v$ is differentiable at $x_{0}$, we need to show that the previous inequality is indeed an equality. Assume, by contradiction, that $v_{-}^{\prime}\left(x_{0}\right)<v_{+}^{\prime}\left(x_{0}\right)$. Then we can construct a sequence of functions $\left\{\varphi_{n}\right\}_{n \in \mathbb{N}} \subset$ $C^{2}\left(\mathbb{R}_{++}\right)$such that, for every $n \in \mathbb{N}$,

$$
\varphi_{n}\left(x_{0}\right)=v\left(x_{0}\right), \quad \varphi_{n} \leq v, \quad \varphi_{n}^{\prime}\left(x_{0}\right)=\frac{v_{-}^{\prime}\left(x_{0}\right)+v_{+}^{\prime}\left(x_{0}\right)}{2}, \quad \varphi_{n}^{\prime \prime}\left(x_{0}\right) \geq n .
$$

Then $\mathscr{L} \varphi_{n}\left(x_{0}\right)-f\left(x_{0}\right) \rightarrow-\infty$ as $n \rightarrow \infty$, which is impossible as $v$ is a viscosity supersolution to (QVI), by Proposition 4.5. Hence it must be $v_{-}^{\prime}\left(x_{0}\right)=v_{+}^{\prime}\left(x_{0}\right)$. By arbitrariness of $x_{0}$, this shows that $v$ is differentiable on $\mathbb{R}_{++}$. By semiconvexity we deduce that $v \in C^{1}\left(\mathbb{R}_{++}\right)$(see [65, Theorem 25.5]).

The fact that $v \in C^{2}(\mathscr{C} ; \mathbb{R})$ follows from a standard localization argument: in each interval $(a, b) \subset \mathscr{C}$ the function $v$ is a viscosity solution to the linear equation $\mathscr{L} u-f=0$ with boundary conditions $u(a)=v(a)$ and $u(b)=v(b)$. By uniform ellipticity of $\mathscr{L}$ over $(a, b)$ (see, e.g., [36, Ch. 6]), this equation admits a unique solution in $C^{2}((a, b) ; \mathbb{R})$, which must also be a viscosity solution. By uniqueness of viscosity solutions to the linear equation above with Dirichlet boundary conditions, we conclude that $v$ coincide with the classical solution, hence $v \in C^{2}((a, b) ; \mathbb{R})$. As $\mathscr{C}$ is open, the claim follows by arbitrariness of $(a, b)$. 
Corollary 4.7. We have

(i) $v^{\prime}(x+\zeta)=c_{0}$, for every $x \in \mathscr{A}, \forall \zeta \in \Xi(x)$.

(ii) $v^{\prime}(x)=c_{0}$, for every $x \in \mathscr{A}$.

Proof. The proof is the same as in [43, Lemma. 5.2] and we skip it for the sake of brevity.

Corollary 4.7(i) will be used in the next section to characterize the optimal target point, i.e. the point in the continuation region where it is optimal to place the system when it reaches the action region.

\section{Explicit expression of the value function}

In this section we characterize $\mathscr{C}, \mathscr{A}$, and $v$ up to the decreasing solution of the homogeneous ODE $\mathscr{L}=0$ and to the solution of a nonlinear system of three algebraic equations.

Lemma 5.1. $\mathscr{A}$ does not contain any interval of the form $[a, \infty)$, with a $>0$. In particular $\mathscr{C} \neq \varnothing$.

Proof. Assume, by contradiction, that there exists $a>0$ such that $\mathscr{A} \supset[a, \infty)$. Then, due to Lemma 4.7(ii), we have

$$
v(x)=c_{0}(x-a)+v(a), \quad \forall x \geq a,
$$

which contradicts Proposition 3.2. On the other hand we should also have

$$
v(x)=\mathscr{M} v(x), \quad \forall x \geq a .
$$

So it must be

$$
c_{0}(x-a)+v(a)=\sup _{i>0}\left\{c_{0}(x+i-a)+v(a)-c_{0} i-c_{1}\right\} \quad \forall x \geq a,
$$

which is impossible as $c_{1}>0$.

The following assumption ensures that the action region is an interval.

Assumption 5.2. $\left.b\right|_{\mathbb{R}_{+}}$is concave.

Lemma 5.3. Let Assumption 5.2 hold. Then $\mathscr{A}$ is an interval.

Proof. Since $\mathscr{A}$ is closed, it is sufficient to show that there do not exist points $x_{0}, x_{1} \in \mathbb{R}_{++}$, with $x_{0}<x_{1}$, such that $x_{0}, x_{1} \in \mathscr{A}$ and $\left(x_{0}, x_{1}\right) \subset \mathscr{C}$. Arguing by contradiction, we assume that such points instead exist. Given $x \in\left(x_{0}, x_{1}\right)$, set $j:=i-\left(x_{1}-x\right)$ for every $i>0$. Then, recalling that $x \in \mathscr{C}$, so $v(x)>\mathscr{M} v(x)$, and that $x_{1} \in \mathscr{A}$, hence $v\left(x_{1}\right)=\mathscr{M} v\left(x_{1}\right)$, we can write

$$
\begin{aligned}
v(x) & >\mathscr{M} v(x)=\sup _{i>0}\left\{v(x+i)-c_{0} i-c_{1}\right\} \geq \sup _{i>x_{1}-x}\left\{v(x+i)-c_{0} i-c_{1}\right\} \\
& =\sup _{j>0}\left\{v\left(x_{1}+j\right)-c_{0} j-c_{1}\right\}+c_{0}\left(x-x_{1}\right)=v\left(x_{1}\right)+c_{0}\left(x-x_{1}\right), \quad \forall x \in\left(x_{0}, x_{1}\right) .
\end{aligned}
$$

Therefore

$$
v(x)-v\left(x_{1}\right)>c_{0}\left(x-x_{1}\right) \quad \forall x \in\left(x_{0}, x_{1}\right) .
$$

Due to Proposition 4.2(i), we have for some for some $y_{1}>x_{1}, y_{1} \in \mathscr{C}$,

$$
v\left(x_{1}\right)=v\left(y_{1}\right)-c_{0}\left(y_{1}-x_{1}\right)-c_{1} .
$$


On the other hand, $v \geq \mathscr{M} v$ implies

$$
v(x) \geq v\left(y_{1}\right)-c_{0}\left(y_{1}-x\right)-c_{1} \quad \forall x \in\left(x_{1}, y_{1}\right) .
$$

Combining (5.2) and (5.3) we get

$$
v(x)-v\left(x_{1}\right) \geq c_{0}\left(x-x_{1}\right) \quad \forall x \in\left(x_{1}, y_{1}\right) .
$$

Then (5.1) and (5.4) show that the function

$$
\varphi(x)=v\left(x_{1}\right)+c_{0}\left(x-x_{1}\right), \quad x \in \mathbb{R}_{++},
$$

is such that $\varphi\left(x_{1}\right)=v\left(x_{1}\right)$ and $v-\varphi$ has a local minimum at $x_{1}$. Since $v$ is a viscosity supersolution to (QVI), this implies

$$
\rho v\left(x_{1}\right)-c_{0} b\left(x_{1}\right) \geq f\left(x_{1}\right) .
$$

Now, by (5.1), there exists $\xi \in\left(x_{0}, x_{1}\right)$ such that $v^{\prime}(\xi)<c_{0}$. Let

$$
y_{2}:=\sup \left\{x \in\left[x_{0}, \xi\right): v^{\prime}(x) \geq c_{0}\right\} .
$$

The definition above is well posed as $x_{0} \in \mathscr{A}$, so that by Corollary 4.7(ii) we have $v^{\prime}\left(x_{0}\right)=c_{0}$. Moreover, by continuity of $v^{\prime}$ and by definition of $y_{2}$ we have

$$
y_{2}<\xi<x_{1}, \quad v^{\prime}\left(y_{2}\right)=c_{0}, \quad v^{\prime}(x)<c_{0} \quad \forall x \in\left(y_{2}, \xi\right) .
$$

Therefore, considering that $v$ is twice differentiable in $\left(x_{0}, \xi\right)$ as this interval is contained in $\mathscr{C}$, from (5.6) and by continuity of $v^{\prime}$ we see that

$$
v^{\prime}\left(y_{2}\right)=c_{0}, v^{\prime \prime}\left(y_{2}\right) \leq 0 .
$$

The equality $\mathscr{L} v=f$ holds in classical sense at $y_{2}$, hence (5.7) entails

$$
\rho v\left(y_{2}\right)-c_{0} b\left(y_{2}\right) \leq f\left(y_{2}\right) .
$$

Combining (5.5) with (5.8), we get

$$
\rho\left(v\left(x_{1}\right)-v\left(y_{2}\right)\right)-c_{0}\left(b\left(x_{1}\right)-b\left(y_{2}\right)\right) \geq f\left(x_{1}\right)-f\left(y_{2}\right) .
$$

On the other hand, considering (5.1) with $x=y_{2}$, and then combining it with (5.9), we get

$$
\rho c_{0}\left(x_{1}-y_{2}\right)-c_{0}\left(b\left(x_{1}\right)-b\left(y_{2}\right)\right)>f\left(x_{1}\right)-f\left(y_{2}\right)
$$

Now, as $x_{1} \in \mathscr{A}$, by (5.2) we have

$$
v\left(y_{1}\right)-c_{0}\left(y_{1}-x_{1}\right)-c_{1}=\sup _{y>x_{1}}\left\{v(y)-c_{0}\left(y-x_{1}\right)-c_{1}\right\} .
$$

The function $v$ is twice differentiable at $y_{1}$ since $y_{1} \in \mathscr{C}$, so (5.11) yields

$$
v^{\prime}\left(y_{1}\right)=c_{0}, v^{\prime \prime}\left(y_{1}\right) \leq 0 .
$$

Therefore the equality $\mathscr{L} v\left(y_{1}\right)=f\left(y_{1}\right)$ yields the inequality

$$
\rho v\left(y_{1}\right)-c_{0} b\left(y_{1}\right) \leq f\left(y_{1}\right) .
$$


Combining (5.12) with (5.5), we get

$$
\rho\left(v\left(y_{1}\right)-v\left(x_{1}\right)\right)-c_{0}\left(b\left(y_{1}\right)-b\left(x_{1}\right)\right) \leq f\left(y_{1}\right)-f\left(x_{1}\right) .
$$

On the other hand, from (5.11) we get

$$
v\left(y_{1}\right)-v\left(x_{1}\right) \geq c_{0}\left(y_{1}-x_{1}\right) .
$$

So, from (5.13) and (5.14) we get

$$
\rho c_{0}\left(y_{1}-x_{1}\right)-c_{0}\left(b\left(y_{1}\right)-b\left(x_{1}\right)\right) \leq f\left(y_{1}\right)-f\left(x_{1}\right) .
$$

To conclude, note that (5.10) and (5.15) are not compatible with the strict concavity of

$$
\mathbb{R}_{++} \rightarrow \mathbb{R}, x \mapsto f(x)+c_{0} b(x)-\rho c_{0} x
$$

which follows from Assumptions 2.3 and 5.2.

Under Assumption 5.2, Lemma 5.1 and Lemma 5.3 provide

$$
\begin{aligned}
\text { either (i) } & \mathscr{C}=\mathbb{R}_{++} \\
\text {or (ii) } & \exists r, s, 0 \leq r<s<\infty: \mathscr{C}=(0, r) \cup(s, \infty) .
\end{aligned}
$$

Case (i) above corresponds to the case in which the continuation region invades all the state space and it is never convenent to undertake an action. In case (ii) the action region is not empty and there is convenience to undertake an action when the system reaches this region.

Consider the homogeneous ODE

$$
\mathscr{L} u=0 \quad \text { on } \mathbb{R}_{++} .
$$

By [19, Th. 16.69] its general solution is of the form

$$
u=A \psi+B \varphi, \quad A, B \in \mathbb{R},
$$

where $\psi, \varphi$ are, respectively, the unique (up to a multiplicative constant) strictly increasing and strictly decreasing solutions to (5.17) and, as 0 and $\infty$ are not accessible boundaries for the reference diffusion $Z$, these fundamental solutions satifsy the following boundary conditions

$$
\psi\left(0^{+}\right):=\lim _{x \rightarrow 0^{+}} \psi(x)=0, \quad \varphi\left(0^{+}\right):=\lim _{x \rightarrow 0^{+}} \varphi(x)=+\infty, \quad \lim _{x \rightarrow \infty} \psi(x)=+\infty, \quad \lim _{x \rightarrow \infty} \varphi(x)=0 .
$$

Other properties of these functions can be found on [19, Sec. 16.11]. On the other hand, the function $\hat{v}$ defined in (3.2) is the unique solution in $\mathbb{R}_{++}$, within the class of functions having at most linear growth, to the nonhomogeneous ODE $\mathscr{L} u=f$ (see [19, Th. 16.72]: actually in the quoted result the function $f$ is required to be bounded, but the proof works as well in our context within the class of functions having at most linear growth). It follows that every classical solution to

$$
\mathscr{L} u=f \text {, over } \mathscr{I} \subset \mathbb{R}_{++},
$$

where $\mathscr{I}$ is an open interval, must have the form $u=A \psi+B \varphi+\hat{v}$. Therefore, as by Proposition 4.5 and Theorem 4.6 the value function $v$ solves in classical sense (5.19), according to the two possibilities of (5.16), in case (i) there must exist real numbers $A, B$ such that

$$
v=\hat{v}+A \psi+B \varphi \text { on } \quad \mathbb{R}_{++} ;
$$

in case (ii) there must exist real numbers $A_{r}, B_{r}, A_{s}, B_{s}$

$$
\left\{\begin{array}{lll}
v=\hat{v}+A_{r} \psi+B_{r} \varphi & \text { on } & (0, r), \\
v=\hat{v}+A_{s} \psi+B_{s} \varphi & \text { on } & (s, \infty) .
\end{array}\right.
$$


Proposition 5.4. Let Assumption 5.2 hold. According to the cases (i) and (ii) of (5.16) we have, respectively:

- if case (i) holds, then $v \equiv \hat{v}$, hence $A=B=0$ in (5.20);

- if case (ii) holds, then $\lim _{x \rightarrow \infty}(v(x)-\hat{v}(x))=0$ and $A_{s}=B_{r}=0, A_{r}, B_{s} \geq 0$ in (5.21).

Proof. Assume that case (i) holds. As $\mathscr{L} v=f$ on $\mathscr{C}=\mathbb{R}_{++}$, by a standard localization procedure we get (see, e.g., the proof of Proposition 3.2)

$$
v(x)=\mathbb{E}\left[\int_{0}^{t} e^{-\rho s} f\left(X_{s}^{x, \phi}\right) d s\right]+\mathbb{E}\left[e^{-\rho t} v\left(X_{t}^{x, \phi}\right)\right] \quad \forall t \in \mathbb{R}_{+} .
$$

We pass to the limit $t \rightarrow \infty$ on the first addend of the right hand side by using the monotone convergence theorem. As for the second addend, we use (3.4) and (3.7) with $I=\varnothing$ to write

$$
0 \leq \mathbb{E}\left[e^{-\rho t} v\left(X_{t}^{x, \varnothing}\right)\right] \leq e^{-\rho t} \frac{f^{*}(\alpha)}{\rho}+\frac{\alpha}{\rho} x \quad \forall \alpha \in\left(0, c_{0} \rho\right] .
$$

Then

$$
0 \leq \limsup _{t \rightarrow \infty} \mathbb{E}\left[e^{-\rho t} v\left(X_{t}^{x, \varnothing}\right)\right] \leq \frac{\alpha}{\rho} x \quad \forall \alpha \in\left(0, c_{0} \rho\right] .
$$

By arbitrariness of $\alpha$ we conclude that

$$
\lim _{t \rightarrow \infty} \mathbb{E}\left[e^{-\rho t} v\left(X_{t}^{x, \varnothing}\right)\right]=0 .
$$

Hence

$$
v(x)=\mathbb{E}\left[\int_{0}^{\infty} e^{-\rho s} f\left(X_{s}^{x, \phi}\right) d s\right] .
$$

By definition of $\hat{v}$ and by the inequality $v \geq \hat{v}$, this proves the claim.

Now assume that case (ii) holds. For each $x>s$ set $\tau_{x}:=\inf \left\{t \geq 0: X_{t}^{x, \varnothing} \leq s\right\}$. As $\infty$ is a natural boundary for $Z^{0, x}=X^{x, \varnothing}$, by (A.2) we have

$$
\lim _{x \rightarrow \infty} \mathbb{P}\left\{\tau_{x} \geq M\right\}=1 \quad \forall M>0 .
$$

If $0<x<x^{\prime}$, by (2.7) with $I=\varnothing$ we get

$$
\text { P-a.s., } X_{t}^{x, \varnothing} \leq X_{t}^{x^{\prime}, \varnothing} \text { for all } t \geq 0,
$$

so, we also have $\tau_{x} \leq \tau_{x^{\prime}} \mathbb{P}$-a.s.. If $\left\{x_{n}\right\}_{n \in \mathbb{N}}$ is a sequence diverging to $\infty$, we then have

$$
\lim _{n \rightarrow \infty} \tau_{x_{n}}=\infty \quad \mathbb{P} \text {-a.s.. }
$$

As $\mathscr{L} v=f$ on $(s, \infty)$, as for (5.22), we get

$$
v\left(x_{n}\right)=\mathbb{E}\left[\int_{0}^{\tau_{x_{n}} \wedge t} e^{-\rho \zeta} f\left(X_{\zeta}^{x_{n}, \varnothing}\right) d \zeta\right]+\mathbb{E}\left[e^{-\rho\left(\tau_{x_{n}} \wedge t\right)} v\left(X_{t \wedge \tau_{x_{n}}}^{x_{n}, \varnothing}\right)\right] \quad \forall t \in \mathbb{R}_{+}, n \in \mathbb{N} .
$$

Therefore, splitting over $\left\{\tau_{x_{n}}<t\right\}$ and $\left\{\tau_{x_{n}} \geq t\right\}$ the second addend on the right hand side,

$$
\begin{aligned}
v\left(x_{n}\right) & =\mathbb{E}\left[\int_{0}^{\tau_{x_{n}} \wedge t} e^{-\rho \zeta} f\left(X_{\zeta}^{x_{n}, \varnothing}\right) d \zeta\right]+\mathbb{E}\left[\mathbf{1}_{\left\{\tau_{x_{n}} \geq t\right\}} e^{-\rho t} v\left(X_{t}^{x_{n}, \varnothing}\right)\right]+\mathbb{E}\left[\mathbf{1}_{\left\{\tau_{x_{n}}<t\right\}} e^{-\rho\left(\tau_{x_{n}} \wedge t\right)} v\left(X_{t \wedge \tau_{x_{n}}}^{x_{n}, \varnothing}\right)\right] \\
& \leq \mathbb{E}\left[\int_{0}^{\tau_{x_{n}} \wedge t} e^{-\rho \zeta} f\left(X_{\zeta}^{x_{n}, \varnothing}\right) d \zeta\right]+\mathbb{E}\left[\mathbf{1}_{\left\{\tau_{x_{n}} \geq t\right\}} e^{-\rho t} v\left(X_{t}^{x_{n}, \varnothing}\right)\right]+\mathbb{E}\left[e^{-\rho \tau_{x_{n}}} \mathbf{1}_{\left\{\tau_{x_{n}}<t\right\}}\right] v(s) .
\end{aligned}
$$


for all $t \geq 0$. Now we pass to the limit $t \rightarrow \infty$ by using the same arguments used to obtain (5.23), and we get

$$
v\left(x_{n}\right) \leq \mathbb{E}\left[\int_{0}^{\tau_{x_{n}}} e^{-\rho \zeta} f\left(X_{\zeta}^{x_{n}, \varnothing}\right) d \zeta\right]+\mathbb{E}\left[e^{-\rho \tau_{x_{n}}} \mathbf{1}_{\left\{\tau_{x_{n}}<\infty\right\}}\right] v(s) .
$$

Then, the definition of $\hat{v}$ provides

$$
v\left(x_{n}\right)-\mathbb{E}\left[e^{-\rho \tau_{x_{n}}} \mathbf{1}_{\left\{\tau_{x_{n}}<\infty\right\}}\right] v(s) \leq \hat{v}\left(x_{n}\right)-\mathbb{E}\left[\mathbf{1}_{\left\{\tau_{x_{n}}<\infty\right\}} \int_{\tau_{x_{n}}}^{\infty} e^{-\rho \zeta} f\left(X_{\zeta}^{x_{n}, \varnothing}\right) d \zeta\right] \leq \hat{v}\left(x_{n}\right) .
$$

Using (5.25) and recalling that $v \geq \hat{v}$, we conclude $\lim _{n \rightarrow \infty}\left(v\left(x_{n}\right)-\hat{v}\left(x_{n}\right)\right)=0$. Since the sequence $\left\{x_{n}\right\}_{n \in \mathbb{N}}$ was arbitrary, we conclude

$$
\lim _{x \rightarrow \infty}(v(x)-\hat{v}(x))=0 .
$$

From (5.18) and (5.27) we have $A_{s}=0$ and $B_{s} \geq 0$. Finally, since $v \geq \hat{v}$ and $v$ is finite in $(0, r)$, from (5.18) we have $A_{r} \geq 0$ and $B_{r}=0$.

Set

$$
\hat{v}^{*}(z):=\sup _{x>0}\{\hat{v}(x)-z x\}, \quad z \in \mathbb{R}_{++} .
$$

We are going to introduce an assumption, requiring that $c_{1}$ is not too large, that guarantees, at once, that the action region is not empty and that the structure of the continuation and action regions are

$$
\mathscr{A}=(0, s] \text { and } \mathscr{C}=(s, \infty) \text { for some } s>0 .
$$

Under this nice structure, it turns out that it is convenient to undertake an action when the system lies below a given threshold and lat it evolve autonomously when the system lies above this threshold. Henceforth, we will call this threshold trigger boundary.

Assumption 5.5. $c_{1}<\hat{v}^{*}\left(c_{0}\right)$.

The following result provides a way to check explicitly the validity of Assumption 5.5.

Proposition 5.6. Let $f(x) \geq K x^{\gamma}$ for some $K>0, \gamma \in(0,1)$, and set $K^{\prime}:=\frac{\gamma K}{\rho+\gamma L_{b}+\frac{1}{2} \gamma(1-\gamma) L_{\sigma}^{2}}$. Then

$$
\hat{v}^{*}\left(c_{0}\right)=K^{\prime} \frac{1-\gamma}{\gamma}\left(\frac{c_{0}}{K^{\prime}}\right)^{\frac{\gamma}{\gamma-1}}
$$

Proof. Let $x \in \mathbb{R}_{++}$. With a localization procedure similar to the one of the proof of Proposition 3.2, we get from Itô's formula

$$
\begin{aligned}
& \mathbb{E}\left[e^{-\rho t}\left|X_{t}^{x, \varnothing}\right|^{\gamma}\right] \\
& =x^{\gamma}+\mathbb{E}\left[\int_{0}^{t} e^{-\rho s}\left[-\rho\left(X_{s}^{x, \varnothing}\right)^{\gamma}+\gamma\left(X_{s}^{x, \varnothing}\right)^{\gamma-1} b\left(X_{s}^{x, \varnothing}\right)+\frac{1}{2} \gamma(\gamma-1)\left(X_{s}^{x, \varnothing}\right)^{\gamma-2} \sigma^{2}\left(X_{s}^{x, \varnothing}\right)\right] d s\right] \\
& \geq x^{\gamma}+\mathbb{E}\left[\int_{0}^{t} e^{-\rho s}\left[-\rho\left(X_{s}^{x, \varnothing}\right)^{\gamma}-L_{b}(1-\gamma)\left(X_{s}^{x, \varnothing}\right)^{\gamma}-\frac{1}{2} L_{\sigma}^{2} \gamma(1-\gamma)\left(X_{s}^{x, \varnothing}\right)^{\gamma}\right] d s\right] .
\end{aligned}
$$

Then we get

$$
\mathbb{E}\left[e^{-\rho t}\left(X_{t}^{x, \varnothing}\right)^{\gamma}\right] \geq x^{\gamma} e^{-\left(\rho+\gamma L_{b}+\frac{1}{2} \gamma(1-\gamma) L_{\sigma}^{2}\right) t}, \quad \forall t \in \mathbb{R}_{+} .
$$


From that and from the assumption on $f$, we obtain

$$
\hat{v}(x) \geq \frac{K}{\rho+\gamma L_{b}+\frac{1}{2} \gamma(1-\gamma) L_{\sigma}^{2}} x^{\gamma}=\frac{K^{\prime}}{\gamma} x^{\gamma}, \quad \forall x \in \mathbb{R}_{++} .
$$

Hence,

$$
\hat{v}^{*}\left(c_{0}\right):=\sup _{x>0}\left\{\hat{v}(x)-c_{0} x\right\} \geq \sup _{x>0}\left\{\frac{K^{\prime}}{\gamma} x^{\gamma}-c_{0} x\right\}=K^{\prime} \frac{1-\gamma}{\gamma}\left(\frac{c_{0}}{K^{\prime}}\right)^{\frac{\gamma}{\gamma-1}}
$$

Proposition 5.7. Let Assumptions 5.2 and 5.5 hold. Then there exists $s>0$ such that $\mathscr{C}=(s, \infty)$ and, consequently, $\mathscr{A}=(0, s]$.

Proof. First, notice that, as $\hat{v}$ satisfies (3.4), it follows that $\hat{v}^{*}$ is finite on $\mathbb{R}_{++}$. Considering that $v \geq \hat{v}$ and that $\hat{v}$ is nondecreasing, we have

$$
\begin{aligned}
\lim _{x \rightarrow 0^{+}} v(x) & \geq \lim _{x \rightarrow 0^{+}} \mathscr{M} v(x) \geq \lim _{x \rightarrow 0^{+}} \mathscr{M} \hat{v}(x)=\lim _{x \rightarrow 0^{+}} \sup _{i>0}\left\{\hat{v}(x+i)-c_{0} i-c_{1}\right\} \\
& \geq \lim _{x \rightarrow 0^{+}} \sup _{i>0}\left\{\hat{v}(i)-c_{0} i-c_{1}\right\}=\hat{v}^{*}\left(c_{0}\right)-c_{1}>0 .
\end{aligned}
$$

Now assume by contradiction that $(0, r) \subset \mathscr{C}$, for some $r>0$. By Proposition 5.4 we have

$$
v(x)=\hat{v}(x)+A_{r} \psi(x), \quad x \in(0, r)
$$

for some $A_{r} \geq 0$. Then, as $\psi\left(0^{+}\right)=0$, we must have $v\left(0^{+}\right)=\hat{v}\left(0^{+}\right)=0$. The latter contradicts (5.28), hence we conclude.

Under Assumptions 5.2 and 5.5, the structure of $\mathscr{C}$ and $\mathscr{A}$ established by Proposition 5.7 joined with Proposition 5.4 provides the following structure for $v$ : for some $B=B_{s} \geq 0$

$$
v(x)= \begin{cases}B \varphi(x)+\hat{v}(x), & \text { if } x \in(s, \infty) \\ B \varphi(s)+\hat{v}(s)-c_{0}(s-x), & \text { if } x \in(0, s]\end{cases}
$$

Lemma 5.8. Let Assumption 5.2 hold. Let $a \geq 0$ and let $u \in C^{2}((a, \infty) ; \mathbb{R})$ satisfy $\mathscr{L} u=f$ on $(a, \infty)$. If $x_{0} \in(a, \infty)$ is a local minimum point for $u^{\prime}$, then $u^{\prime}\left(x_{0}\right)>0$ and there is no local maximum point for $u^{\prime}$ in $\left(x_{0}, \infty\right)$.

Proof. As $b, \sigma, f \in C^{1}\left(\mathbb{R}_{++} ; \mathbb{R}\right)$, from

$$
\rho u(x)=b(x) u^{\prime}(x)+\frac{1}{2} \sigma^{2}(x) u^{\prime \prime}(x)+f(x), \quad \forall x \in(a, \infty),
$$

we obtain $u^{\prime \prime} \in C^{1}((a, \infty)$; $\mathbb{R})$, i.e. $u \in C^{3}((a, \infty) ; \mathbb{R})$. We differentiate (5.30) getting

$$
\rho u^{\prime}(x)=b^{\prime}(x) u^{\prime}(x)+b(x) u^{\prime \prime}(x)+\frac{1}{2} \sigma^{2}(x) u^{\prime \prime \prime}(x)+\sigma \sigma^{\prime}(x) u^{\prime \prime}(x)+f^{\prime}(x), \quad \forall x \in(a, \infty) .
$$

Let $x_{0} \in(a, \infty)$ be a local minimum point for $u^{\prime}$. Then $u^{\prime \prime}\left(x_{0}\right)=0$ and $u^{\prime \prime \prime}\left(x_{0}\right) \geq 0$ so, by (5.31), we have

$$
\rho u^{\prime}\left(x_{0}\right) \geq b^{\prime}\left(x_{0}\right) u^{\prime}\left(x_{0}\right)+f^{\prime}\left(x_{0}\right) .
$$


Note that from (5.32), using Assumptions 2.3 and 2.4, we obtain $u^{\prime}\left(x_{0}\right)>0$. Now, arguing by contradiction, assume that $x_{1} \in\left(x_{0}, \infty\right)$ is local maximum point for $u^{\prime}$. Then $u^{\prime \prime}\left(x_{1}\right)=0$ and $u^{\prime \prime \prime}\left(x_{1}\right) \leq 0$, so, by (5.31), we have

$$
\rho u^{\prime}\left(x_{1}\right) \leq b^{\prime}\left(x_{1}\right) u^{\prime}\left(x_{1}\right)+f^{\prime}\left(x_{1}\right) .
$$

Without loss of generality, we can assume that

$$
u^{\prime}\left(x_{0}\right) \leq u^{\prime}\left(x_{1}\right)
$$

Combining (5.32) and (5.33) and taking account that $f^{\prime}$ is strictly decreasing, we get

$$
\left(\rho-b^{\prime}\left(x_{1}\right)\right) u^{\prime}\left(x_{1}\right) \leq f^{\prime}\left(x_{1}\right)<f^{\prime}\left(x_{0}\right) \leq\left(\rho-b^{\prime}\left(x_{0}\right)\right) u^{\prime}\left(x_{0}\right) .
$$

Now, by Assumption 5.2 we have $b^{\prime}\left(x_{0}\right) \geq b^{\prime}\left(x_{1}\right)$. So, the fact that $u^{\prime}\left(x_{0}\right)>0$ and (5.35) yield

$$
\left(\rho-b^{\prime}\left(x_{1}\right)\right) u^{\prime}\left(x_{1}\right)<\left(\rho-b^{\prime}\left(x_{1}\right)\right) u^{\prime}\left(x_{0}\right) .
$$

By Assumption 2.4, we have the $\rho-b^{\prime}\left(x_{1}\right)>0$. Hence, from (5.36) we obtain $u^{\prime}\left(x_{1}\right)<u^{\prime}\left(x_{0}\right)$, contradicting (5.34).

Recall that a function $\varphi: \mathscr{O} \rightarrow \mathbb{R}$, with $\mathscr{O}$ open interval, is said quasiconcave if

$$
\varphi\left(\lambda x+(1-\lambda) x^{\prime}\right)>\min \left\{\varphi(x), \varphi\left(x^{\prime}\right)\right\} \quad \forall x, x^{\prime} \in \mathscr{O}, \forall \lambda \in(0,1) .
$$

Strictly quasiconcave functions can be characterized as functions that are either strictly increasing, or strictly decreasing, or strictly increasing on the left of a point $x^{*} \in \mathscr{O}$ and strictly decreasing on the right of $x^{*}$.

Lemma 5.9. Let Assumption 5.2 hold. Let $a \geq 0$, let $u \in C^{2}((a, \infty) ; \mathbb{R})$ satisfy $\mathscr{L} u=f$ on $(a, \infty)$, and assume that $\liminf _{x \rightarrow \infty} u^{\prime}(x) \leq 0$. Then $u^{\prime}$ is strictly quasiconcave.

Proof. By virtue of [9, Proposition 3.24], it is sufficient to show that $u^{\prime}$ does not admit any local minimum. Argue by contradiction and assume that $x_{0} \in(a, \infty)$ is a local minimum point for $u^{\prime}$. The proof of Lemma 5.8 shows then that $u^{\prime}\left(x_{0}\right)>0$. Hence, since $\liminf _{x \rightarrow \infty} u^{\prime}(x) \leq 0$, there must exists a local maximum point $x_{1} \in\left(x_{0}, \infty\right)$. This contradicts Lemma 5.8 and we conclude.

Proposition 5.10. Let Assumptions 5.2 and 5.5 hold.

(i) There exists a unique $S \in \mathscr{C}=(s, \infty)$ such that $v^{\prime}(S)=c_{0}$.

(ii) There exists (a unique) $x^{*} \in(s, S)$ such that $v^{\prime}$ is strictly increasing in $\left(s, x^{*}\right]$ and strictly decreasing in $\left[x^{*}, \infty\right)$.

(iii) $\lim _{x \rightarrow \infty} v^{\prime}(x)=0$.

Proof. (i) Corollary 4.7(i) and Proposition 4.2(i) yield the existence of $S \in \mathscr{C}=(s, \infty)$ such that $v^{\prime}(S)=c_{0}$. Regarding uniqueness, observe first that $v$ satisfies the requirements of Lemma 5.9 (plugging $v$ in place of $u$ ) with $a=s$ and where

$$
\liminf _{x \rightarrow \infty} v^{\prime}(x) \leq 0
$$


holds by (3.4). Then the fact that $v^{\prime}(s)=c_{0}$ by Corollary 4.7(ii) yields the uniqueness.

(ii) By (5.29) we have $v^{\prime}(s)=c_{0}$. By (i) above we have $v^{\prime}(S)=c_{0}$ and $v^{\prime}(x) \neq c_{0}$ for each $x \in(s, S)$. Then the claim follows by Lemma 5.9 .

(iii) This follows immediately by monotonicity of $v^{\prime}$ on $\left[x^{*},+\infty\right)$, (5.37), and Proposition 3.1, which provides $v^{\prime} \geq 0$.

Theorem 5.11. Let Assumptions 5.2 and 5.5 hold. The value function has the form

$$
v(x)= \begin{cases}B \varphi(x)+\hat{v}(x), & \text { if } x \in(s, \infty), \\ B \varphi(S)+\hat{v}(S)-c_{0}(S-x)-c_{1}, & \text { if } x \in(0, s],\end{cases}
$$

and the triple $(B, s, S)$ is the unique solution in $\mathbb{R}_{+} \times \mathbb{R}_{++}^{2}$ to the system

$$
\left\{\begin{array}{l}
\text { (i) } \quad B \varphi(s)+\hat{v}(s)=B \varphi(S)+\hat{v}(S)-c_{0}(S-s)-c_{1}, \\
\text { (ii) } B \varphi^{\prime}(s)+\hat{v}^{\prime}(s)=c_{0}, \\
\text { (iii) } B \varphi^{\prime}(S)+\hat{v}^{\prime}(S)=c_{0} .
\end{array}\right.
$$

Proof. Consider (5.29). The expression of $v$ over $(s, \infty)$ in (5.38) and (5.29) is the same. As for the expression of $v$ over $(0, s]$, we note that, by definition of $\Xi(s)$, Proposition 4.2, Corollary 4.7, and Proposition 5.10(i), we have

$$
0<S-s=\underset{i>0}{\operatorname{argmax}}\left\{v(s+i)-c_{0} i-c_{1}\right\} .
$$

Since $s \in \mathscr{A}$, we have $v(s)=[\mathscr{M} v](s)$; so, from (5.40) we get

$$
v(s)=v(S)-c_{0}(S-s)-c_{1},
$$

from which we get the expression of $v$ over $(0, s]$ in (5.38). Then the three equations of (5.39) follow, respectively, by imposing the continuity of $v$ at $s$, the smooth-fit at $s$ (as $v \in C^{1}\left(\mathbb{R}_{++} ; \mathbb{R}\right)$ ), and the condition of Proposition 5.10(i) defining $S$.

To show that (5.39) has a unique solution in $\mathbb{R}_{+} \times \mathbb{R}_{++}^{2}$, we consider the function

$$
h(\hat{B}, x)=\hat{B} \varphi(x)+\hat{v}(x), \quad(\hat{B}, x) \in \mathbb{R}_{++} \times \mathbb{R}_{++} .
$$

For each $\hat{B} \geq 0, \mathscr{L} h(\hat{B}, \cdot)=0$ in $\mathbb{R}_{++}$and $\liminf _{x \rightarrow \infty} h_{x}(\hat{B}, x) \leq 0$ by (3.4) and (5.18). By Lemma 5.9 $h_{x}(\hat{B}, \cdot)$ is strictly quasiconcave; hence, there exist at most two solutions $\hat{s}, \hat{S}$ to $h_{x}(\hat{B}, \cdot)=c_{0}$ in $\mathbb{R}_{++}$. If such solutions exist, we have $h(\hat{B}, \cdot)-c_{0}>0$ on $(\hat{s} \wedge \hat{S}, \hat{s} \vee \hat{S})$. Therefore, if $(\hat{B}, \hat{s}, \hat{S}) \in \mathbb{R}_{+} \times \mathbb{R}_{++}^{2}$ solves (5.39), then (5.39)(i) yields

$$
0<c_{1}=[\hat{B} \varphi(\hat{S})+\hat{v}(\hat{S})]-[\hat{B} \varphi(\hat{s})+\hat{v}(\hat{s})]-c_{s}(\hat{S}-\hat{s})=\int_{\hat{s}}^{\hat{S}}\left(h_{x}(\hat{B}, r)-c_{0}\right) d r .
$$

This forces $\hat{s}=\hat{s} \wedge \hat{S}, \hat{S}=\hat{s} \vee \hat{S}, \hat{s} \neq \hat{S}$. By the argument above we see that, if $\left(B_{1}, s_{1}, S_{1}\right)$ and $\left(B_{2}, s_{2}, S_{2}\right)$ are two different solutions to (5.39) in $\mathbb{R}_{+} \times \mathbb{R}_{++}^{2}$, we need to have $s_{1}<S_{1}, s_{2}<S_{2}$, and $B_{1} \neq B_{2}$.

Now assume, by contradiction, that $\left(B_{1}, s_{1}, S_{1}\right)$ and $\left(B_{2}, s_{2}, S_{2}\right)$ are two different solutions of (5.39) in $\mathbb{R}_{+} \times \mathbb{R}_{++}^{2}$. Without loss of generality, we can assume $B_{1}<B_{2}$. Recalling that $\varphi$ is strictly decreasing, we have

$$
h_{x}\left(B_{1}, \cdot\right)>h_{x}\left(B_{2}, \cdot\right) .
$$


The latter inequality, Lemma 5.9, and (5.39)(ii)-(iii) provide

$$
\left(s_{1}, S_{1}\right) \supset\left(s_{2}, S_{2}\right), \quad h_{x}\left(B_{1}, \cdot\right)-c_{0}>0 \text { on }\left(s_{1}, S_{1}\right) .
$$

We can then write, using (5.41)-(5.42) and (5.39)(i),

$$
\begin{aligned}
0 & =c_{1}-c_{1}=\left(h\left(B_{1}, S_{1}\right)-h\left(B_{1}, s_{1}\right)-c_{0}\left(S_{1}-s_{1}\right)\right)-\left(h\left(B_{2}, S_{2}\right)-h\left(B_{2}, s_{2}\right)-c_{0}\left(S_{2}-s_{2}\right)\right) \\
& =\int_{s_{1}}^{S_{1}}\left(h_{x}\left(B_{1}, \xi\right)-c_{0}\right) d \xi-\int_{s_{2}}^{S_{2}}\left(h_{x}\left(B_{2}, \xi\right)-c_{0}\right) d \xi \\
& \geq \int_{s_{2}}^{S_{2}}\left(h_{x}\left(B_{1}, \xi\right)-h_{x}\left(B_{2}, \xi\right)\right) d \xi>0
\end{aligned}
$$

which is a contradiction.

\section{Optimal control}

In this section, through Theorem 6.1, we describe the structure of an optimal control for our problem through a recursive rule. In the economic literature - see the stream of papers on stochastic impulse control at the beginning of the paragraph on the related linterature in the Introduction and [16] - this rule is known as $(S, s)$-rule. Informally, this rule, rigorously stated in Theorem 6.1 below, can be described as follows.

- The point $s$ works as an optimal trigger boundary: when the state variable is at level $s$ or below such level (i.e., it is within the action region $\mathscr{A}$ ), the controller acts.

- The point $S$ works as an optimal target boundary: when the controller acts, she/he does that in such a way to place the state variable at the level $S \in \mathscr{C}$.

- When the state variable lies in the region $\mathscr{C}$, the controller let it evolve autonomously without undertaking any action until it exits from this region.

Such rule is made rigorous by the following construction. Let $x \in \mathbb{R}_{++}$and consider the control $I^{*}=\left\{\left(\tau_{n}, i_{n}\right)\right\}_{n \geq 1}$ defined as follows:

$$
\left\{\begin{array}{l}
\tau_{1}:= \begin{cases}0 & \text { if } x \leq s, \\
\inf \left\{t \geq 0: Z_{t}^{0, x} \leq s\right\} & \text { if } x>s,\end{cases} \\
i_{1}:= \begin{cases}S-x & \text { if } \left.\tau_{1}=0 \text { (i.e. } x \leq 0\right), \\
S-s & \text { if } \left.\tau_{1}>0 \text { (i.e. } x>s\right),\end{cases}
\end{array}\right.
$$

and then, recursively for $n \geq 1$,

$$
\left\{\begin{array}{l}
\tau_{n+1}:= \begin{cases}\tau_{n}+\inf \left\{t>0: Z_{\tau_{n}+t}^{\tau_{n}, S} \leq s\right\} & \text { if } \tau_{n}<\infty \\
\infty & \text { otherwise }\end{cases} \\
i_{n+1}:=S-s
\end{array}\right.
$$

Note that, for $\mathbb{P}$-a.e. $\omega \in\left\{\tau_{n}<\infty\right\}$, by continuity of $\mathbb{R}_{+} \rightarrow \mathbb{R}, t \mapsto Z_{\tau_{n}+t}^{\tau_{n}, S}(\omega)$ and since $S>s$, we have $\tau_{n+1}(\omega)>\tau_{n}(\omega)$. 
Theorem 6.1 (Optimal control). Let Assumptions 5.2 and 5.5 hold. Let $x \in \mathbb{R}_{++}$and consider the control $I^{*}=\left\{\left(\tau_{n}, i_{n}\right)\right\}_{n \geq 1}$ defined above. Then $I^{*} \in \mathscr{I}$ and it is optimal for the problem starting at $x$, i.e., $J\left(x, I^{*}\right)=v(x)$.

Proof. Admissibility. As noticed above, $\tau_{n}<\tau_{n+1} \mathbb{P}$-a.s. on $\left\{\tau_{n}<\infty\right\}$. Moreover, for each $n \geq 1, i_{n}$ is constant; so, as a random variable, it is trivially $\mathscr{F}_{\tau_{n}}$-measurable.

Now, for fixed $\varepsilon>0$ such that $S-\varepsilon S^{2}>s$, define the auxiliary sequence $\left\{\tau_{n}^{\varepsilon}\right\}_{n \geq 1}$ of stopping times by

$$
\tau_{1}^{\varepsilon}:= \begin{cases}0 & \text { if } x \leq s \\ \inf \left\{t \geq 0: Z_{t}^{0, x}-\varepsilon\left(Z_{t}^{0, x}+t\right)^{2} \leq s\right\} & \text { if } x>s\end{cases}
$$

and

$$
\tau_{n+1}^{\varepsilon}:=\tau_{n}^{\varepsilon}+\inf \left\{t \geq 0: Z_{\tau_{n}^{\varepsilon}+t}^{\tau_{n}^{\varepsilon}, S}-\varepsilon\left(Z_{\tau_{n}^{\varepsilon}+t}^{\tau_{n}^{\varepsilon}, S}+t\right)^{2} \leq s\right\} \text { for } n \geq 1 .
$$

We notice that $\tau_{n}^{\varepsilon}$ is finite and $\tau_{n+1}^{\varepsilon}>\tau_{n}^{\varepsilon}$ P-a.s.. Moreover, the random variables $\left\{\tau_{n+1}^{\varepsilon}-\tau_{n}^{\varepsilon}\right\}_{n \geq 1}$ are identically distributed and $\tau_{n+1}^{\varepsilon}-\tau_{n}^{\varepsilon}$ is independent on $\mathscr{F}_{\tau_{n}^{\varepsilon}}$. Finally, it can be verified by induction that

$$
\lim _{\varepsilon \rightarrow 0^{+}} \tau_{n}^{\varepsilon}=\tau_{n} \quad \mathbb{P} \text {-a.s. on }\left\{\tau_{n}<\infty\right\}
$$

from which we obtain

$$
\liminf _{\varepsilon \rightarrow 0^{+}} e^{-\rho \tau_{n}^{\varepsilon}} \geq e^{-\rho \tau_{n}} \quad \mathbb{P} \text {-a.s.. }
$$

Define $Y^{\varepsilon}:=\inf \left\{t \geq 0: Z_{t}^{0, S}-\varepsilon\left(Z_{t}^{0, S}+t\right)^{2} \leq s\right\}$. Then $\tau_{n+1}^{\varepsilon}-\tau_{n}^{\varepsilon} \sim Y^{\varepsilon}$ for all $n \geq 1$. Observe that $Y^{\varepsilon}$ increases as $\varepsilon$ tends to $0^{+}$. Let $Y:=\lim _{\varepsilon \rightarrow 0^{+}} Y^{\varepsilon}$. Since $S-\varepsilon S^{2}>s$ entails $Y^{\varepsilon}>0$, we have in particular $Y>0$. We can then write, using (6.1) and Fatou's Lemma in the first inequality below,

$$
\begin{aligned}
\mathbb{E}\left[e^{-\rho \tau_{n+1}}\right] & \leq \liminf _{\varepsilon \rightarrow 0^{+}} \mathbb{E}\left[e^{-\rho \tau_{n+1}^{\varepsilon}}\right]=\liminf _{\varepsilon \rightarrow 0^{+}} \mathbb{E}\left[e^{-\rho\left(\tau_{n+1}^{\varepsilon}-\tau_{n}^{\varepsilon}\right)} e^{-\rho \tau_{n}^{\varepsilon}}\right] \\
& =\liminf _{\varepsilon \rightarrow 0^{+}} \mathbb{E}\left[\mathbb{E}\left[e^{-\rho\left(\tau_{n+1}^{\varepsilon}-\tau_{n}^{\varepsilon}\right)} e^{-\rho \tau_{n}^{\varepsilon}} \mid \mathscr{F}_{\tau_{n}^{\varepsilon}}\right]\right]=\liminf _{\varepsilon \rightarrow 0^{+}}\left(\mathbb{E}\left[e^{-\rho\left(\tau_{n+1}^{\varepsilon}-\tau_{n}^{\varepsilon}\right)}\right] \mathbb{E}\left[e^{-\rho \tau_{n}^{\varepsilon}}\right]\right) \\
& =\liminf _{\varepsilon \rightarrow 0^{+}}\left(\mathbb{E}\left[e^{-\rho Y^{\varepsilon}}\right] \mathbb{E}\left[e^{-\rho \tau_{n}^{\varepsilon}}\right]\right) \stackrel{(\text { by induction) }}{=} \liminf _{\varepsilon \rightarrow 0^{+}}\left(\mathbb{E}\left[e^{-\rho Y^{\varepsilon}}\right]\right)^{n} \mathbb{E}\left[e^{-\rho \tau_{1}^{\varepsilon}}\right] \\
& \leq\left(\mathbb{E}\left[e^{-\rho Y}\right]\right)^{n} .
\end{aligned}
$$

Summing over $n \geq 1$ and taking into account that $\mathbb{E}\left[e^{-\rho Y}\right]<1$, from (6.2) we get

$$
\mathbb{E}\left[\sum_{n \geq 1} e^{-\rho \tau_{n+1}}\right]<\infty
$$

Both conditions (2.3) and (2.4) follow from (6.3), so the control $I^{*}$ is admissible.

Optimality. Set $X^{*}:=X^{x, I^{*}}$. We observe that, by (3.4), (3.7), and (6.3), we have

$$
\lim _{T \rightarrow \infty} \mathbb{E}\left[e^{-\rho T} v\left(X_{T}^{*}\right)\right]=0 .
$$

Let $T>0$ and set $\tau_{0}:=0^{-}$. Observe that by definition $X^{*} \in[s,+\infty)$ and recall that $\mathscr{L} v=f$ on $\mathscr{C}=(s, \infty)$. For all $n \in \mathbb{N}$ we apply Itô's formula to $v\left(X^{*}\right)$ in the interval $\left[\tau_{n} \wedge T, \tau_{n+1} \wedge T\right)$. Note that $v^{\prime}$ is bounded in $[s, \infty)$ by Proposition 5.10 , so

$$
\mathbb{E}\left[\int_{\tau_{n} \wedge T}^{\tau_{n+1} \wedge T} v^{\prime}\left(X_{t}^{*}\right) d W_{t}\right]=0 \quad \forall n \in \mathbb{N} .
$$


Hence, taking the expectation in the Itô formula and taking into account that $\mathscr{L} v\left(X^{*}\right)=f\left(X^{*}\right)$, we get

$$
\mathbb{E}\left[e^{-\rho\left(\tau_{n+1} \wedge T\right)} v\left(X_{\left(\tau_{n+1} \wedge T\right)^{-}}^{*}\right)\right]-\mathbb{E}\left[e^{-\rho\left(\tau_{n} \wedge T\right)} v\left(X_{\tau_{n} \wedge T}^{*}\right)\right]=-\mathbb{E}\left[\int_{\tau_{n} \wedge T}^{\tau_{n+1} \wedge T} e^{-\rho t} f\left(X_{t}^{*}\right) d t\right], \quad \forall n \in \mathbb{N} .
$$

Now fix for the moment $\omega \in \Omega, n \geq 1$, and assume that $\tau_{n}(\omega) \leq T$. By definition of $i_{n}(\omega)$ and considering that $X_{\tau_{n}^{-}}^{*}(\omega) \in \mathscr{A}$ we have (cf. also Corollary 4.7, Proposition 4.2(i), and the definition of $S$ in Proposition 5.10(i))

$$
i_{n}(\omega)=\underset{i>0}{\operatorname{argmax}}\left\{v\left(X_{\tau_{n}^{-}}^{*}(\omega)+i\right)-c_{0} i-c_{1}\right\} .
$$

Hence, considering that $\mathscr{M} v\left(X_{\tau_{n}^{-}}^{*}(\omega)\right)=v\left(X_{\tau_{n}^{-}}^{*}(\omega)\right)$, we have

$$
e^{-\rho \tau_{n}(\omega)} v\left(X_{\tau_{n}(\omega)}^{*}\right)-e^{-\rho \tau_{n}} v\left(X_{\tau_{n}(\omega)^{-}}^{*}\right)=e^{-\rho \tau_{n}(\omega)}\left(c_{0} i_{n}(\omega)+c_{1}\right) .
$$

It follows that, for all $n \geq 1$,

$$
\begin{aligned}
\mathbb{E}\left[e ^ { - \rho ( \tau _ { n } \wedge T ) } \left(v\left(X_{\tau_{n} \wedge T}^{*}\right)\right.\right. & \left.-v\left(X_{\left(\tau_{n} \wedge T\right)^{-}}^{*}\right)\right]= \\
& =\mathbb{E}\left[e^{-\rho\left(\tau_{n} \wedge T\right)}\left(v\left(X_{T}^{*}\right)-v\left(X_{T^{-}}^{*}\right)\right) \mathbf{1}_{\left\{\tau_{n}>T\right\}}\right]+\mathbb{E}\left[e^{-\rho \tau_{n}}\left(c_{0} i_{n}+c_{1}\right) \mathbf{1}_{\left\{\tau_{n} \leq T\right\}}\right] .
\end{aligned}
$$

Using (6.5) and (6.7), we can then write, for $N \geq 1$,

$$
\begin{aligned}
& \mathbb{E}\left[e^{-\rho\left(\tau_{N+1} \wedge T\right)} v\left(X_{\tau_{N+1} \wedge T}^{*}\right)\right]-v(x)=\sum_{n=0}^{N} \mathbb{E}\left[e^{-\rho\left(\tau_{n+1} \wedge T\right)} v\left(X_{\tau_{n+1} \wedge T}^{*}\right)-e^{-\rho\left(\tau_{n} \wedge T\right)} v\left(X_{\tau_{n} \wedge T}^{*}\right)\right] \\
& =\sum_{n=0}^{N} \mathbb{E}\left[e^{-\rho\left(\tau_{n+1} \wedge T\right)}\left(v\left(X_{\tau_{n+1} \wedge T}^{*}\right)-v\left(X_{\left(\tau_{n+1} \wedge T\right)^{-}}^{*}\right)\right)\right] \\
& +\sum_{n=0}^{N} \mathbb{E}\left[e^{-\rho\left(\tau_{n+1} \wedge T\right)} v\left(X_{\left(\tau_{n+1} \wedge T\right)^{-}}^{*}\right)-e^{-\rho\left(\tau_{n} \wedge T\right)} v\left(X_{\tau_{n} \wedge T}^{*}\right)\right] \\
& =\sum_{n=0}^{N}\left(\mathbb{E}\left[e^{-\rho\left(\tau_{n+1} \wedge T\right)}\left(v\left(X_{T}^{*}\right)-v\left(X_{T^{-}}^{*}\right)\right) \mathbf{1}_{\left\{\tau_{n+1}>T\right\}}\right]+\mathbb{E}\left[e^{-\rho \tau_{n+1}}\left(c_{0} i_{n+1}+c_{1}\right) \mathbf{1}_{\left\{\tau_{n+1} \leq T\right\}}\right]\right) \\
& -\sum_{n=0}^{N} \mathbb{E}\left[\int_{\tau_{n} \wedge T}^{\tau_{n+1} \wedge T} e^{-\rho t} f\left(X_{t}^{*}\right) d t\right] .
\end{aligned}
$$

By passing to the limit $N \rightarrow \infty$ and using (2.3), we obtain

$$
\begin{aligned}
& \mathbb{E}\left[e^{-\rho T} v\left(X_{T}^{*}\right)\right]-v(x)+\mathbb{E}\left[\int_{0}^{T} e^{-\rho t} f\left(X_{t}^{*}\right) d t\right] \\
= & \sum_{n=0}^{\infty}\left(\mathbb{E}\left[e^{-\rho\left(\tau_{n+1} \wedge T\right)}\left(v\left(X_{T}^{*}\right)-v\left(X_{T^{-}}^{*}\right)\right) \mathbf{1}_{\left\{\tau_{n+1}>T\right\}}\right]+\mathbb{E}\left[e^{\left.\left.-\rho \tau_{n+1}\left(c_{0} i_{n+1}+c_{1}\right) \mathbf{1}_{\left\{\tau_{n+1} \leq T\right\}}\right]\right) .}\right.\right.
\end{aligned}
$$

We take now the $\liminf _{T \rightarrow \infty}$ using (6.4) on the first addend of the left hand side, monotone convergence on the third addend of the left hand side, and Fatou's lemma on the right hand side. We obtain

$$
-v(x)+\mathbb{E}\left[\int_{0}^{\infty} e^{-\rho t} f\left(X_{t}^{*}\right) d t\right] \geq \sum_{n=0}^{\infty} \mathbb{E}\left[e^{-\rho \tau_{n+1}}\left(c_{0} i_{n+1}+c_{1}\right)\right],
$$

which shows that $I^{*}$ is optimal. 


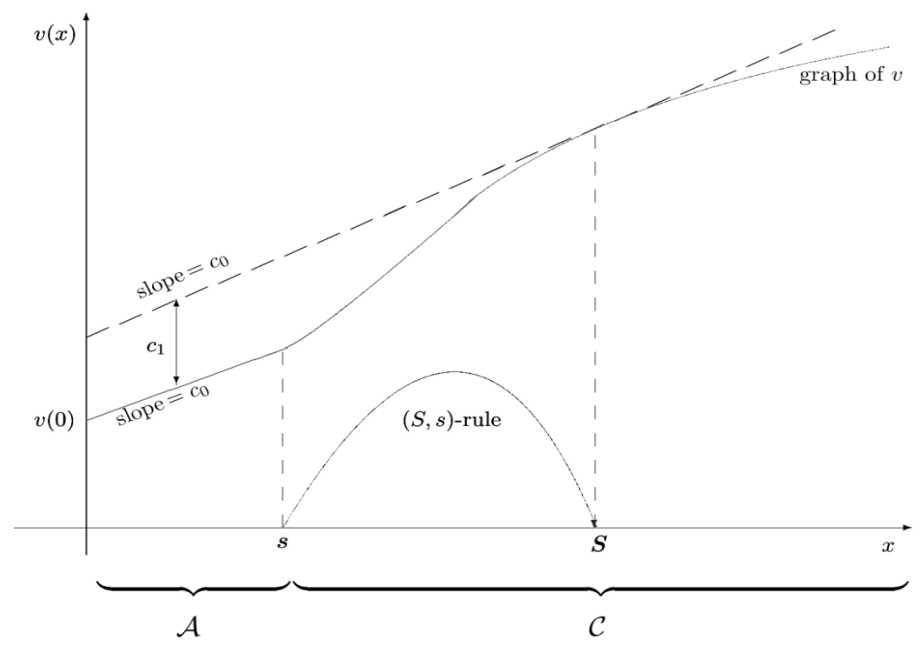

Figure 1: An illustrative picture of the value function and of the $S-s$ rule.

\section{Numerical illustration in the linear case}

In the previous sections we have characterized the solution of the dynamic optimization problem through the unique solution of the nonlinear algebraic system (5.39) in the triple $(A, s, S)$. In this section we specialize the study when the reference process $Z$ follows a geometric Brownian motion dynamics, i.e. when $b(x):=v x, \sigma(x):=\sigma x$, with $v \in \mathbb{R}, \sigma>0$, and when $f(x)=\frac{x^{\gamma}}{\gamma}$, with $0<\gamma<1$, assuming

$$
\rho>v^{+} \text {. }
$$

In this way, Assumptions 2.1, 5.2, 2.4, 2.3, 3.3(ii)-(iv) are satisfied $\left({ }^{7}\right)$. In the present case we have

$$
\varphi(x)=x^{m},
$$

where $m$ is the negative root of the characteristic equation

$$
\rho-v m-\frac{1}{2} \sigma^{2} m(m-1)=0
$$

associated with $\mathscr{L} u=0$, i.e.

$$
m=\left(\frac{1}{2}-\frac{v}{\sigma^{2}}\right)-\sqrt{\left(\frac{1}{2}-\frac{v}{\sigma^{2}}\right)^{2}+\frac{2 \rho}{\sigma^{2}}}
$$

and

$$
\hat{v}(x)=C_{\gamma} \frac{x^{\gamma}}{\gamma}, \quad C_{\gamma}:=\left(\rho-v \gamma+\frac{1}{2} \gamma(1-\gamma) \sigma^{2}\right)^{-1} .
$$

The problem with no fixed cost, i.e. when $c_{1}=0$, is investigated in the singular control setting (the right one to get existence of optimal controls, see Remark 2.5) in [63, Sec. 4.5]. In this case, the

\footnotetext{
${ }^{7}$ Actually, we should consider $b(x)=v x$ if $x>0$ and $b(x)=0$ otherwise, and similarly for $\sigma$, in order to fit Assumption 2.1. But this does not matter because our controlled process lies in $\mathbb{R}_{++}$.
} 
value function $v$ and the optimal reflection boundary $s$ are characterized in [63, Th. 4.5.7] through an algebraic system too. Such system can be solved providing, in our notation,

$$
s=\left(\frac{c_{0}(m-1)}{C_{\gamma}(m-\gamma)}\right)^{\frac{1}{\gamma-1}}, \quad B=\frac{C_{\gamma}(1-\gamma)}{m(m-1)} s^{\gamma-m} .
$$

We make Assumption 5.5; the latter in the present case reads as

$$
c_{1}<C_{\gamma}^{\frac{1}{1-\gamma}} c_{0}^{\frac{\gamma}{1-\gamma}}\left(\frac{1}{\gamma}-1\right) .
$$

Moreover, Assumption 3.3(i) would read as

$$
\rho>\max \left\{4|v|+6 \sigma^{2}, 2|v|+2 \sigma^{2}\right\}=4|v|+6 \sigma^{2} .
$$

However, as we show below, in the linear-homogeneous case under consideration here, we do not need to make this assumption: we can exploit the linear dependence of the controlled process on the initial datum and the homogeneity of $f$ to show the result of semiconvexity stated, for the general case, in Proposition 3.7. Consequently, the other results of the paper hold under no further assumption. Indeed, observing that the terms $\left\{i_{n}\right\}_{n \geq 1}$ enter in the dynamics of $X^{x, I}$ in additive form, we have

$$
X_{t}^{x, I}-X_{t}^{y, I}=X_{t}^{x, \varnothing}-X_{t}^{y, \varnothing}=(x-y) e^{\left(v-\frac{\sigma^{2}}{2}\right) t+\sigma W_{t}}, \quad \forall I \in \mathscr{I}, \forall x, y \in \mathbb{R}_{++},
$$

that we can use to prove the following result.

Proposition 7.1. In the above framework we have, for every $\lambda \in[0,1]$, and every $x, y \geq \varepsilon>0$

$$
v(\lambda x+(1-\lambda) y)-\lambda v(x)-(1-\lambda) v(y) \leq \lambda(1-\lambda)(1-\gamma) C_{\gamma}^{-1} \varepsilon^{\gamma-2}(y-x)^{2} .
$$

Proof. Let $0<\xi \leq \xi^{\prime}$. Then, for suitable $\eta, \eta^{\prime} \in\left[\xi, \xi^{\prime}\right]$ we have, by Lagrange's Theorem,

$$
\begin{aligned}
f\left(\lambda \xi+(1-\lambda) \xi^{\prime}\right)-\lambda f(\xi)- & (1-\lambda) f\left(\xi^{\prime}\right)= \\
& =-\lambda\left[f(\xi)-f\left(\xi+(1-\lambda)\left(\xi^{\prime}-\xi\right)\right)\right]-(1-\lambda)\left[f\left(\xi^{\prime}\right)-f\left(\xi^{\prime}+\lambda\left(\xi-\xi^{\prime}\right)\right)\right] \\
& =\lambda(1-\lambda) f^{\prime}(\eta)\left(\xi^{\prime}-\xi\right)-\lambda(1-\lambda) f^{\prime}\left(\eta^{\prime}\right)\left(\xi^{\prime}-\xi\right) \\
& =\lambda(1-\lambda)\left(f^{\prime}(\eta)-f^{\prime}\left(\eta^{\prime}\right)\right)\left(\xi^{\prime}-\xi\right) \\
& \leq \lambda(1-\lambda)\left|f^{\prime \prime}(\xi)\right|\left(\xi^{\prime}-\xi\right)^{2} \\
& =\lambda(1-\lambda)(1-\gamma) \xi^{\gamma-2}\left(\xi^{\prime}-\xi\right)^{2} .
\end{aligned}
$$

Let now $0<\varepsilon \leq x \leq y, \lambda \in[0,1]$, and set $z:=\lambda x+(1-\lambda) y$. Let $\delta>0$ and let $I_{\delta} \in \mathscr{I}$ be a $\delta$-optimal control for $v(z)$. Then, using (7.7), the fact that $X^{x, I} \geq X^{x, \varnothing}$, and recalling (7.6), we get

$$
\begin{aligned}
v(\lambda x+(1-\lambda) y)-\delta-\lambda v(x)-(1-\lambda) v(y) \leq J\left(z, I_{\delta}\right)-\lambda J\left(x, I_{\delta}\right)-(1-\lambda) J\left(y, I_{\delta}\right) \\
=\mathbb{E}\left[\int_{0}^{+\infty} e^{-\rho t}\left(f\left(X_{t}^{z, I_{\delta}}\right)-\lambda f\left(X_{t}^{x, I_{\delta}}\right)-(1-\lambda) f\left(X_{t}^{y, I_{\delta}}\right)\right) d t\right] \\
\leq \lambda(1-\lambda)(1-\gamma) \mathbb{E}\left[\int_{0}^{+\infty} e^{-\rho t}\left(X_{t}^{x, I_{\delta}}\right)^{\gamma-2}\left(X_{t}^{y, I_{\delta}}-X_{t}^{x, I_{\delta}}\right)^{2} d t\right] \\
\leq \lambda(1-\lambda)(1-\gamma) \mathbb{E}\left[\int_{0}^{+\infty} e^{-\rho t}\left(X_{t}^{x, \varnothing}\right)^{\gamma-2}\left(X_{t}^{y, \varnothing}-X_{t}^{x, \varnothing}\right)^{2} d t\right] \\
=\lambda(1-\lambda)(1-\gamma) C_{\gamma}^{-1} x^{\gamma-2}(y-x)^{2} \leq \lambda(1-\lambda)(1-\gamma) C_{\gamma}^{-1} \varepsilon^{\gamma-2}(y-x)^{2},
\end{aligned}
$$

the claim. 


\subsection{Numerical illustration}

We perform a numerical analysis of the solution solving the nonlinear system (5.39). In Figure 2, we provide the picture of the value function and its derivative when the parameters are set as follows: $\rho=0.08, v=-0.07, \sigma=0.25, c_{0}=1, c_{1}=10, \gamma=0.5$. Solving (5.39) with these entries and with $\varphi(x)=x^{m}$, where $m$ is given by (7.2), yields

$$
(B, s, S)=(97.0479,8.7492,56.9930) .
$$
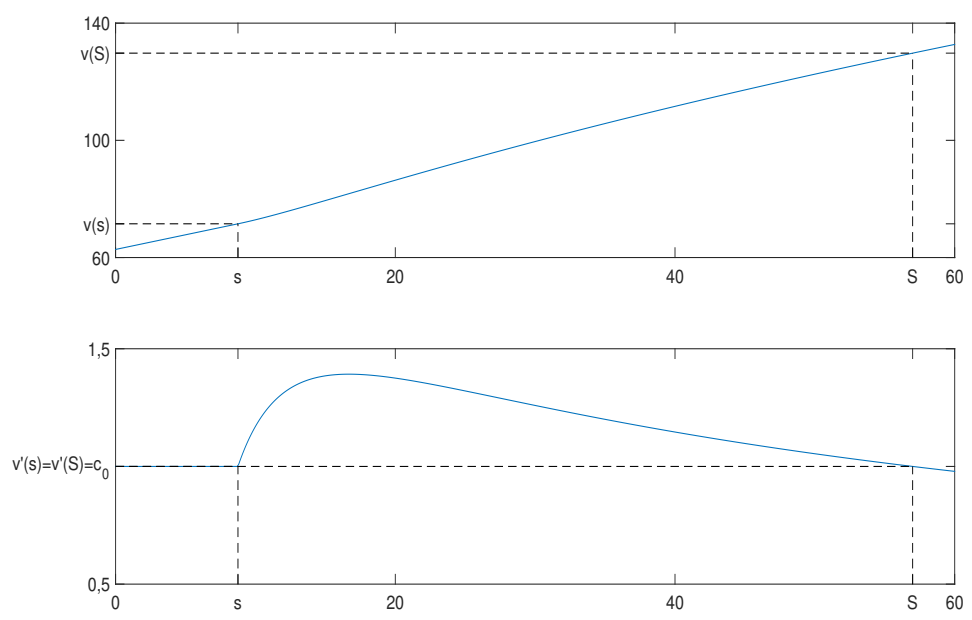

Figure 2: Value function (above) and its derivative (below)

In the rest of this section we discuss numerically the solution, illustrating how changes in parameters affect the value function and the trigger and target boundaries $s, S$, which describe the optimal control $\left({ }^{8}\right)$.

\subsubsection{Impact of volatility}

In Table 1 we report the relevant values the solution for different values of the volatility $\sigma$. The other parameters are set as follows: $\rho=0.08, v=-0.07, \gamma=0.5, c_{0}=1, c_{1}=10$.

Table 1: Solution as function of $\sigma$.

\begin{tabular}{cccccccc}
\hline \hline$\sigma$ & $B$ & $s$ & $S$ & $S-s$ & $v(0)$ & $v(s)$ & $v(S)$ \\
\hline $1 \%$ & 349.2820 & 14.6488 & 69.1073 & 54.4584 & 68.2325 & 82.8813 & 147.3398 \\
$5 \%$ & 313.6460 & 14.2670 & 68.4774 & 54.2104 & 68.0298 & 82.2968 & 146.5072 \\
$10 \%$ & 238.6460 & 13.2168 & 66.6426 & 53.4258 & 67.3856 & 80.6024 & 144.0282 \\
$15 \%$ & 172.6459 & 11.8029 & 63.9264 & 52.1235 & 66.2914 & 78.0943 & 140.2178 \\
$20 \%$ & 126.9781 & 10.2646 & 60.6291 & 50.3644 & 64.7453 & 75.0099 & 135.3743 \\
$25 \%$ & 97.0479 & 8.7492 & 56.9930 & 48.2438 & 62.7645 & 71.5137 & 129.7575 \\
$30 \%$ & 77.1043 & 7.3358 & 53.2006 & 45.8648 & 60.3826 & 67.7184 & 123.5832 \\
\hline
\end{tabular}

Figure 3, drawn imposing the same values of parameters, represents the trigger level $s$, the target

\footnotetext{
8 The simulations are done for negative values of $v$, thinking of it as a depreciation factor. We omit, for the sake of brevity, to report the simulations that we have performed for positive values of $v$, as the outputs show the same qualitative behaviour as in the case of negative $v$.
} 
level $S$, and their difference $S-s$ as functions of the volatility $\sigma$. The figure and the table show that, when uncertainty increases, the action region $\mathscr{A}$ shrinks and the investment size $S-s$ shrinks. The first effect is well-known in the economic literature of irreversible investments without fixed costs as value of waiting to invest: an increase of uncertainty leads to postpone the investment (see [52]). We can see that, in our fixed cost context, also the size of the optimal investment is negatively affected by an increase of uncertainty.
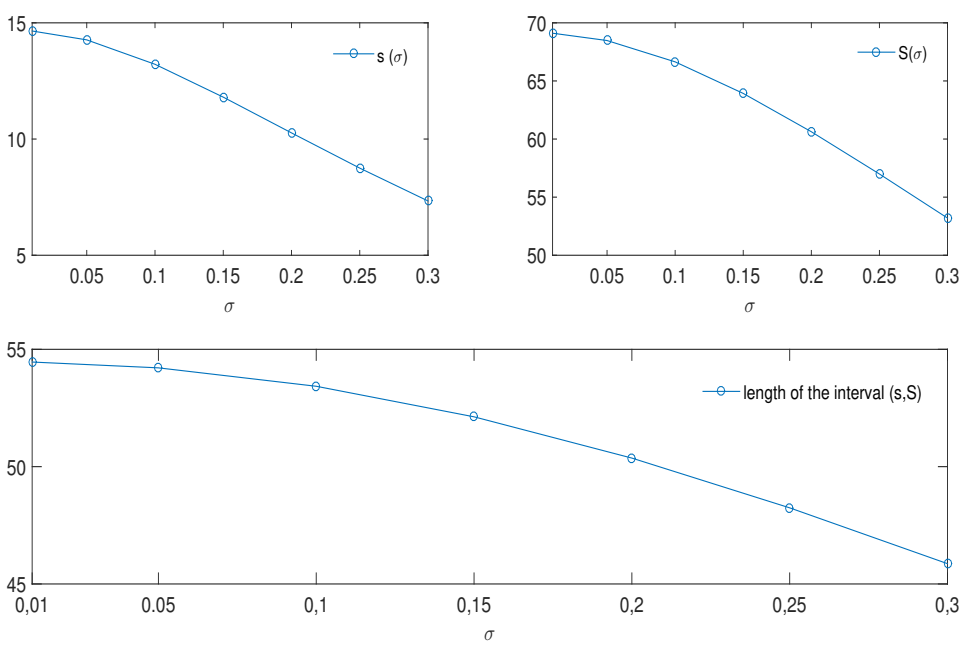

Figure 3: The trigger level $s$, the target level $S$, and the difference $S-s$ as functions of $\sigma$.

\subsubsection{Impact of fixed cost}

In Table 2 we report the relevant values of the solution for different values of the fixed $\operatorname{cost} c_{1}$, when the other parameters are set as follows: $\sigma=0.1, \rho=0.08, v=-0.07, \gamma=0.5, c_{0}=1$. In the row corresponding to $c_{1}=0$, there are reported the outputs of the corresponding singular control problem, computed according to the values of $s$ and $B$ expressed by $(7.4)\left({ }^{9}\right)$. It can be observed that the convergence as $c_{1} \rightarrow 0^{+}$is pretty slow; this is consistent with the theoretical result of [61], which would state, in our case, $\frac{\partial v\left(\cdot ; c_{1}\right)}{\partial c_{1}}\left(0^{+}\right)=-\infty$.

Table 2: Solution as function of $c_{1}$.

\begin{tabular}{lccccccc}
\hline \hline \multicolumn{1}{c}{$c_{1}$} & $B$ & $s$ & $S$ & $S-s$ & $v(0)$ & $v(s)$ & $v(S)$ \\
\hline 0 & 577.5165 & 41.6233 & 41.6233 & 0 & 83.2470 & 124.8703 & 124.8703 \\
0.01 & 573.1240 & 38.6466 & 44.5649 & 5.9182 & 83.1362 & 121.7828 & 127.7110 \\
0.5 & 519.9311 & 30.6195 & 52.1522 & 21.5328 & 81.5607 & 112.1802 & 134.2129 \\
1 & 487.9211 & 27.7903 & 54.7042 & 26.9139 & 80.4620 & 108.2523 & 136.1663 \\
10 & 238.6460 & 13.2168 & 66.6426 & 53.4258 & 67.3856 & 80.6024 & 144.0282 \\
30 & 57.6611 & 4.2696 & 72.3953 & 68.1257 & 44.7847 & 49.0543 & 147.1800 \\
50 & 7.9037 & 1.0275 & 73.7826 & 72.7551 & 24.1040 & 25.1315 & 147.8866 \\
\hline
\end{tabular}

\footnotetext{
${ }^{9}$ In this case the optimal control consists in a reflection policy at a boundary; in other terms the interval $[s, S]$ degenerates in a singleton $\{s\}=\{S\}$.
} 
Figure 4, drawn imposing the same values of parameters, shows that, as $c_{1}$ increases, the action region $\mathscr{A}$ shrinks and the investment size $S-s$ expands. Both these effects are expected: the first one is the counterpart of the value of waiting to invest, now with respect to the fixed cost of investment, rather than with respect to uncertainty; the second one expresses the fact that an increase of the fixed cost leads to invest less often, then to provide a larger investment size when the investment is undertaken.
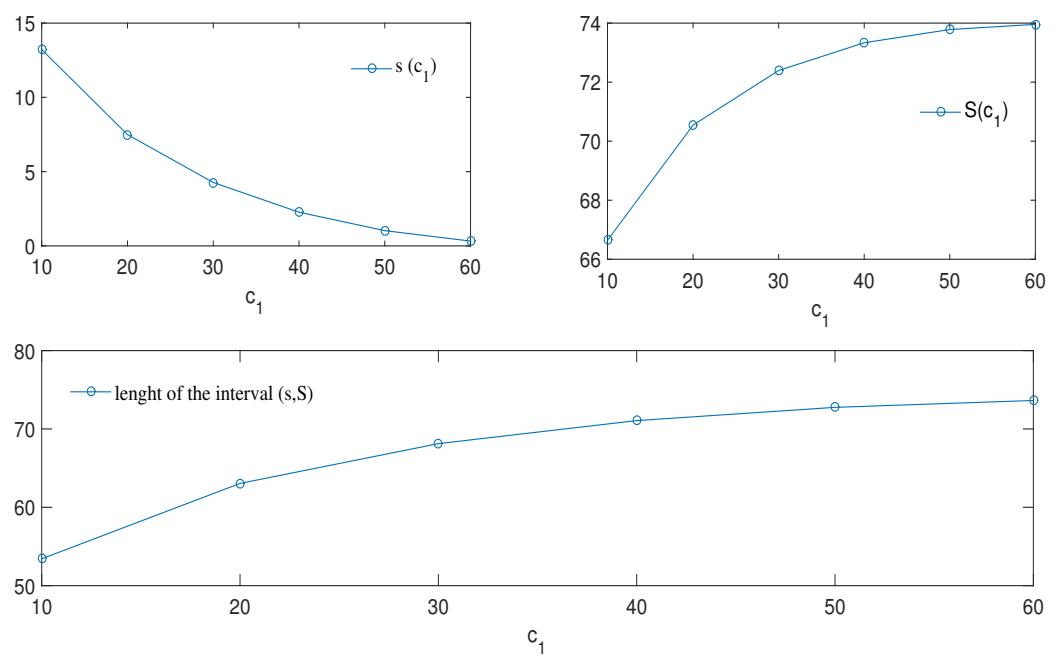

Figure 4: The trigger level $s$, the target level $S$, and the difference $S-s$ as functions of $c_{1}$.

\section{A Appendix}

Proposition A.1. Under Assumption 2.1 the boundaries 0 and $+\infty$ are natural in the sense of Feller's classification for the diffusion $Z^{0, x}$.

Proof. Clearly $+\infty$ is not accessible, in the sense that $Z^{0, x}$ does not explode in finite time. It remains to show that 0 is not accessible, that is

$$
x \in \mathbb{R}_{++} \Longrightarrow Z_{t}^{0, x}>0 \quad \text { P-a.s. } \forall t \geq 0 ;
$$

that both 0 and $+\infty$ are not entrance, that is

$$
\lim _{x \downarrow 0} \mathbb{P}\left\{\tau_{x, y}<t\right\}=0, \quad \lim _{x \uparrow \infty} \mathbb{P}\left\{\tau_{x, y}<t\right\}=0, \quad \forall t, y \in \mathbb{R}_{++} .
$$

To this end, we introduce the speed measure $m$ of the diffusion $Z^{0, x}$ transformed to natural scale (see [19, Prop. 16.81, Th. 16.83]). Up to a multiplicative constant, we have

$$
m(d y)=\frac{2}{\sigma^{2}(y)} e^{\int_{1}^{y} \frac{2 b(\xi)}{\sigma^{2}(\xi)} d \xi} d y, \quad y \in \mathbb{R}_{++} .
$$

Assumption 2.1 implies that for some $C_{0}, C_{1}>0$ we have $|b(\xi)| \leq C_{0} \xi$ and $\sigma^{2}(\xi) \leq C_{1} \xi^{2}$ for every $\xi \in \mathbb{R}_{+}$. According to [19, Prop. 16.43] we compute $\int_{0}^{1} y m(d y)$. We have

$$
\int_{0}^{1} y m(d y) \geq \int_{0}^{1} \frac{2 y}{\sigma^{2}(y)} e^{\int_{1}^{y} \frac{-2 C_{0} \xi}{\sigma^{2}(\xi)} d \xi} d y .
$$


Set $F(y):=\int_{1}^{y} \frac{-2 C_{0} \xi}{\sigma^{2}(\xi)} d \xi$. We have

$$
\begin{aligned}
\int_{0}^{1} \frac{2 y}{\sigma^{2}(y)} e^{\int_{1}^{y} \frac{-2 C_{0} \xi}{\sigma^{2}(\xi)} d \xi} d y & =-\frac{1}{C_{0}} \int_{0}^{1} F^{\prime}(y) e^{F(y)} d y=-\frac{1}{C_{0}}\left[e^{F(1)}-\lim _{y \rightarrow 0^{+}} e^{F(y)}\right] \\
& =-\frac{1}{C_{0}}\left[1-\lim _{y \rightarrow 0^{+}} e^{\int_{1}^{y}-\frac{2 C_{0} \xi}{\sigma^{2}(\xi)} d \xi}\right]=-\frac{1}{C_{0}}\left[1-e^{\lim _{y \rightarrow 0^{+}} \int_{y}^{1} \frac{2 C_{0}}{C_{1} \xi} d \xi}\right]=+\infty .
\end{aligned}
$$

This shows, by [19, Prop. 16.43], that (A.1) holds, The fact that 0 is not-entrance, i.e. that the first limit in (A.2) holds, is then consequence of [19, Prop. 16.45(a)]. Let us show, finally, that also $+\infty$ is not-entrance, i.e. that the second limit in (A.2) holds. In this case, according to [19, Prop. 16.45(b)] we consider $\int_{1}^{+\infty} y m(d y)$ and see, with the same computations as above, that it is equal to $+\infty$. By the aforementioned result we conclude that $+\infty$ is not entrance.

Remark A.2. The property (A.1) can be generalized to the case of random initial data. Let $\tau$ be a (possibly infinite) $\mathbb{F}$-stopping time and let $\xi$ be an $\mathscr{F}_{\tau}$-measurable random variable, clearly we have the equality in law $Z_{t+\tau}^{\tau, \xi}=\left(Z_{t}^{0, x}\right)_{\left.\right|_{x=\xi}}$. By (A.1), it then follows that

$$
\xi \mathscr{F}_{\tau} \text {-measurable random variable, } \xi>0 \mathbb{P} \text {-a.s. } \Longrightarrow Z_{t+\tau}^{\tau, \xi}>0 \mathbb{P} \text {-a.s. on }\{\tau<\infty\}, \forall t \geq 0 .
$$

Lemma A.3. Let $I \in \mathscr{I}, x, y \in \mathbb{R}_{++}$.

(i) We have

$$
\mathbb{E}\left[\left|X_{s}^{x, I}-X_{s}^{y, I}\right|^{4}\right] \leq|x-y|^{4} e^{C_{0} t} \quad \forall t \geq 0
$$

where $C_{0}:=4 L_{b}+6 L_{\sigma}^{2}$.

(ii) For each $\lambda \in[0,1]$ and $x, y \in \mathbb{R}_{++}$, define $z_{\lambda}:=\lambda x+(1-\lambda) y$. Then

$$
\mathbb{E}\left[\left|X_{t}^{z_{\lambda}, I}-\lambda X_{t}^{x, I}-(1-\lambda) X_{t}^{y, I}\right|^{2}\right] \leq A_{0} \lambda^{2}(1-\lambda)^{2}|x-y|^{4} e^{B_{0} t} \quad \forall \lambda \in[0,1], \forall t \geq 0,
$$

where $A_{0}>0$ and $B_{0}:=2 L_{b}+2 L_{\sigma}^{2}+\tilde{L}_{b}$.

Proof. (i) We apply Itô's formula to $\left|X^{x, I}-X^{y, I}\right|^{4}$ and then - after a standar localization procedure with stopping times to let the stochastic integral term be a martingale and all the other expectations be well defined and finite; see e.g. the proof of Proposition 3.2 - we take the expectation. We get, also using Assumption 2.1,

$$
\begin{aligned}
\mathbb{E}\left[\left|X_{t}^{x, I}-X_{t}^{y, I}\right|^{4}\right] & =|x-y|^{4}+4 \mathbb{E} \int_{0}^{t}\left(X_{u}^{x, I}-X_{u}^{y, I}\right)^{3}\left(b\left(X_{u}^{x, I}\right)-b\left(X_{u}^{y, I}\right)\right) d u \\
& +6 \mathbb{E} \int_{0}^{t}\left(X_{u}^{x, I}-X_{u}^{y, I}\right)^{2}\left(\sigma\left(X_{u}^{x, I}\right)-\sigma\left(X_{u}^{y, I}\right)\right)^{2} d u \\
& \leq|x-y|^{4}+\left(4 L_{b}+6 L_{\sigma}^{2}\right) \int_{0}^{t} \mathbb{E}\left[\left|X_{u}^{x, I}-X_{u}^{y, I}\right|^{4}\right] d u .
\end{aligned}
$$

The claim follows by Gronwall's inequality.

(ii) Define $\Sigma^{\lambda, x, y, I}:=\lambda X^{x, I}+(1-\lambda) X^{y, I}$. We apply Itô’s formula to the process $\left(X^{z_{\lambda}, I}-\Sigma^{\lambda, x, y, I}\right)^{2}$ and then - after a standar localization procedure with stopping times to let the stochastic integral 
term be a martingale and all the other expectations are well defined and finite; see e.g. the proof of Proposition 3.2 - take the expectation, obtaining, also using Assumption 2.1,

$$
\begin{aligned}
\mathbb{E}\left[\left(X_{t}^{z_{\lambda}, I}-\Sigma_{t}^{\lambda, x, y, I}\right)^{2}\right]= & 2 \int_{0}^{t} \mathbb{E}\left[\left(X_{u}^{z_{\lambda}, I}-\Sigma_{u}^{\lambda, x, y, I}\right)\left(b\left(X_{u}^{z_{\lambda}, I}\right)-\lambda b\left(X_{u}^{x, I}\right)-(1-\lambda) b\left(X_{u}^{y, I}\right)\right)\right] d u \\
& +\int_{0}^{t} \mathbb{E}\left[\left(\sigma\left(X_{u}^{z_{\lambda}, I}\right)-\lambda \sigma\left(X_{u}^{x, I}\right)-(1-\lambda) \sigma\left(X_{u}^{y, I}\right)\right)^{2}\right] d u \\
\leq & 2 \int_{0}^{t} \mathbb{E}\left[\left|X_{u}^{z_{\lambda}, I}-\Sigma_{u}^{\lambda, x, y, I}\right| \cdot\left|b\left(X_{u}^{z_{\lambda}, I}\right)-b\left(\Sigma_{u}^{\lambda, x, y, I}\right)\right|\right] d u \\
& +2 \int_{0}^{t} \mathbb{E}\left[\left|X_{u}^{z_{\lambda}, I}-\Sigma_{u}^{\lambda, x, y, I}\right| \cdot\left|b\left(\Sigma_{u}^{\lambda, x, y, I}\right)-\lambda b\left(X_{u}^{t, \xi, I}\right)-(1-\lambda) b\left(X_{u}^{t, \xi^{\prime}, I}\right)\right|\right] d u \\
& +2 \int_{0}^{t} \mathbb{E}\left[\left|\sigma\left(X_{u}^{z_{\lambda}, I}\right)-\sigma\left(\Sigma_{u}^{\lambda, x, y, I}\right)\right|^{2}\right] d u \\
& +2 \int_{0}^{t} \mathbb{E}\left[\left|\sigma\left(\Sigma_{u}^{\lambda, x, y, I}\right)-\lambda \sigma\left(X_{u}^{x, I}\right)-(1-\lambda) \sigma\left(X_{u}^{y, I}\right)\right|^{2}\right] d u \\
\leq & 2\left(L_{b}+L_{\sigma}^{2}\right) \int_{0}^{t} \mathbb{E}\left[\left|X_{u}^{z_{\lambda}, I}-\Sigma_{u}^{\lambda, x, y, I}\right|^{2}\right] d u \\
& +2 \int_{0}^{t} \mathbb{E}\left[\left|X_{u}^{z_{\lambda}, I}-\Sigma_{u}^{\lambda, x, y, I}\right| \cdot\left|b\left(\Sigma_{u}^{\lambda, x, y, I}\right)-\lambda b\left(X_{u}^{t, \xi, I}\right)-(1-\lambda) b\left(X_{u}^{t, \xi^{\prime}, I}\right)\right|\right] d u \\
& +2 \int_{0}^{t} \mathbb{E}\left[\left|\sigma\left(\Sigma_{u}^{\lambda, x, y, I}\right)-\lambda \sigma\left(X_{u}^{x, I}\right)-(1-\lambda) \sigma\left(X_{u}^{y, I}\right)\right|^{2}\right] d u .
\end{aligned}
$$

By doing the same computations as in $[72$, p. 188$]$ in order to obtain $[72$, p. 188 , formulae $(4.22)$ and (4.23)], we have

$$
\begin{array}{ll}
\left|b\left(\lambda x^{\prime}+(1-\lambda) x^{\prime \prime}\right)-\lambda b\left(x^{\prime}\right)-(1-\lambda) b\left(x^{\prime \prime}\right)\right| \leq \tilde{L}_{b} \lambda(1-\lambda)\left|x^{\prime}-x^{\prime \prime}\right|^{2} & \forall x^{\prime}, x^{\prime \prime} \in \mathbb{R}_{++}, \\
\left|\sigma\left(\lambda x^{\prime}+(1-\lambda) x^{\prime \prime}\right)-\lambda \sigma\left(x^{\prime}\right)-(1-\lambda) \sigma\left(x^{\prime \prime}\right)\right| \leq \tilde{L}_{\sigma} \lambda(1-\lambda)\left|x^{\prime}-x^{\prime \prime}\right|^{2} & \forall x^{\prime}, x^{\prime \prime} \in \mathbb{R}_{++}
\end{array}
$$

where $\tilde{L}_{b}, \tilde{L}_{\sigma}$ are as in Assumption 2.1. Then, by using (A.7) and (A.8) in (A.6), we get

$$
\begin{aligned}
\mathbb{E}\left[\left|X_{s}^{z_{\lambda}, I}-\Sigma_{s}^{\lambda, x, y, I}\right|^{2}\right] \leq & 2\left(L_{b}+L_{\sigma}^{2}\right) \int_{0}^{t} \mathbb{E}\left[\left|X_{u}^{z_{\lambda}, I}-\Sigma_{u}^{\lambda, x, y, I}\right|^{2}\right] d u \\
& +2 \lambda(1-\lambda) \tilde{L}_{b} \int_{0}^{t} \mathbb{E}\left[\left|X_{u}^{z_{\lambda}, I}-\Sigma_{u}^{\lambda, x, y, I}\right| \cdot\left|X_{u}^{x, I}-X_{u}^{y, I}\right|^{2}\right] d u \\
& +2 \lambda^{2}(1-\lambda)^{2} \tilde{L}_{\sigma}^{2} \int_{0}^{t} \mathbb{E}\left[\left|X_{u}^{x, I}-X_{u}^{y, I}\right|^{4}\right] d u
\end{aligned}
$$

Using the inequality

$$
2 \lambda(1-\lambda) a b \leq a^{2}+\lambda^{2}(1-\lambda)^{2} b^{2} \quad \forall a, b \in \mathbb{R}
$$


and (A.4) into (A.9), we obtain

$$
\begin{aligned}
\mathbb{E}\left[\left|X_{t}^{z_{\lambda}, I}-\Sigma_{t}^{\lambda, x, y, I}\right|^{2}\right] \leq & \left(2 L_{b}+2 L_{\sigma}^{2}+\tilde{L}_{b}\right) \int_{0}^{t} \mathbb{E}\left[\left|X_{u}^{z_{\lambda}, I}-\Sigma_{u}^{\lambda, x, y, I}\right|^{2}\right] d u \\
& +\lambda^{2}(1-\lambda)^{2}\left(\tilde{L}_{b}+2 \tilde{L}_{\sigma}^{2}\right) \int_{0}^{t} \mathbb{E}\left[\left|X_{u}^{x, I}-X_{u}^{y, I}\right|^{4}\right] d u \\
\leq & \left(2 L_{b}+2 L_{\sigma}^{2}+\tilde{L}_{b}\right) \int_{0}^{t} \mathbb{E}\left[\left|X_{u}^{z_{\lambda}, I}-\Sigma_{u}^{\lambda, x, y, I}\right|^{2}\right] d u \\
& +\left(\tilde{L}_{b}+2 \tilde{L}_{\sigma}^{2}\right) \lambda^{2}(1-\lambda)^{2} \int_{0}^{t} e^{C_{0} u}|x-y|^{4} d u \\
\leq & \left(2 L_{b}+2 L_{\sigma}^{2}+\tilde{L}_{b}\right) \int_{0}^{t} \mathbb{E}\left[\left|X_{u}^{z_{\lambda}, I}-\Sigma_{u}^{\lambda, x, y, I}\right|^{2}\right] d u \\
& +\frac{\tilde{L}_{b}+2 \tilde{L}_{\sigma}^{2}}{C_{0}}\left(e^{C_{0} t}-1\right) \lambda^{2}(1-\lambda)^{2}|x-y|^{4},
\end{aligned}
$$

where $C_{0}$ is the constant of (A.4). We conclude by Gronwall's inequality.

\section{References}

[1] A.B. Abel and J.C. Eberly, "Optimal investment with costly reversibility", Review of Economic Studies, Vol. 63, (1996), pp. 581-593.

[2] R.Aïd, S. Federico, H. Pham and B. Villeneuve, "Explicit investment rules with time-to-build and uncertainty”. J. Econ. Dynam. Control 51 (2015), pp. 240-256.

[3] L.H. Alvarez, "A class of solvable impulse control problems", Applied Mathematics and Optimization, Vol. 49 (2004), pp. 265-295.

[4] L.H. Alvarez, "Irreversible capital accumulation under interest rate uncertainty", Mathematical Methods of Operations Research, Vol. 72 (2009), No. 2, pp. 249-271.

[5] L.H. Alvarez, "Optimal capital accumulation under price uncertainty and costly reversibility”, Journal of Economics, Dynamics and Control, Vol. 35 (2011), No. 10, pp. 1769-1788.

[6] L.H. Alvarez and J. Lempa, "On the optimal stochastic impulse control of linear diffusions", SIAM Journal on Control and Optimization, Vol. 47, No. 2 (2008), pp. 703-732.

[7] R.F. Anderson, "Discounted Replacement, Maintenance, and Repair Problems in Reliability", Mathematics of Operations Research, Vol. 19 (1994), Issue 4, pp. 909-945.

[8] K.J. Arrow, T. Harris and J. Marshak, "Optimal inventory policy”, Econometrica, Vol. 19 (1951), No. 3, pp. 250-272.

[9] M. Avriel, W.E. Diewert, S. Schaible and I. Zang, Generalized Concavity, SIAM, Classics in Applied Mathematics 63, 2010.

[10] F.M. Baldursson and I. Karatzas, "Irreversible investment and industry equilibrium", Finance and Stochastics, Vol. 1 (1997), No. 1, pp. 69-89.

[11] P. Bank, "Optimal control under a dynamic fuel constraint", SIAM Journal on Control and Optimization, Vol. 44 (2005), No. 4, pp. 1529-1541. 
[12] Bar-Ilan, A. Sulem, "Explicit Solution of Inventory Problems with Delivery Lags", Mathematics of Operations Research, Vol. 20 (1995), Issue 3, pp. 709-720.

[13] A. Bar-Ilan, A. Sulem and A. Zanello. "Time-to-Build and Capacity Choice", Journal of Economic Dynamics and Control, Vol. 26, pp. 69-98, 2002.

[14] E. Bayraktar, T. Emmerling and J.L. Menaldi, "On the impulse control of jump diffusions", SIAM Journal on Control and Optimization, Vol. 51 (2013), No. 3, pp. 2612-2637.

[15] C. Belak, S. Christensen and F.T. Seifred, "A general verification result for stochastic impulse control problems", SIAM Journal on Control and Optimization, Vol. 55 (2017), No. 2, pp. 627649.

[16] A. Bensoussan and B. Chevalier-Roignant, "Sequential capacity expansion options", Operations Research, Forthcoming.

[17] A. Bensoussan and J.L. Lions, Impulse control and Quasi-variational inequalities, GauthierVillars, Paris, 1984.

[18] A. Bensoussan, J. Liu, J. Yuan, "Singular control and impulse control: a common approach", Discrete and continuous dynamical systems (Series B), Vol. 13 (2010), No. 1, pp. 27-57.

[19] L. Breiman, Probability, Classics in Applied Mathematics, SIAM (1992).

[20] A. Cadenillas, P. Lakner, M. Pinedo, "Optimal Control of a Mean-Reverting Inventory", Operations Research, Vol. 58 (2010), Issue 6, pp. 1697-1710.

[21] A. Cadellinas and F. Zapatero, "Classical and impulse stochastic control of the exchange rate using interest rates and reserves”, Math. Finance, Vol.1̃0, Issue 2, pp. 141-156, 2000.

[22] Y.-S.A. Chen and X. Guo, "Impulse control of multidimensional jump diffusions in finite time horizon”, SIAM J. on Control and Optim., Vol. 51, No. 3, pp. 2638-2663, 2013.

[23] M.B. Chiarolla and U.G. Haussman, "On a stochastic irreversible investment problem", SIAM Journal on Control and Optimization, Vol. 48 (2009), No. 2, 438-462.

[24] M.B. Chiarolla, G. Ferrari. Identifying the Free Boundary of a Stochastic, Irreversible Investment Problem via the Bank-El Karoui Representation Theorem, SIAM Journal on Control and Optimization 52(2) (2014), pp. 1048-1070.

[25] S. Christensen, "On the Solution of General Impulse Control Problems Using Superharmonic Functions", Stochastic Processes and their Applications, Volume 124 (2014), No. 1, pp. 709 729.

[26] S. Christensen, P. Salminen. "Impulse control and expected suprema". Advances in Applied Probability, Vol. 49, Issue 1, pp. 238-257, 2017.

[27] G.M. Constantidinies and S.F. Richard, "Existence of optimal simple policies for discountedcost inventory and cash management in continuous time", Operations Research, Vol. 26, Issue 4, pp. 620-636, 1978.

[28] M.H. Davis, M.A.H. Dempster, S.P. Sethi and D. Vermes, "Optimal capacity expansion under uncertainty”, Advances in Applied Probability, Vol. 19 (1987), pp. 156-176. 
[29] M. Davis, X. Guo and G. Wu, "Impulse control of multidimensional jump diffusions", SIAM Journal on Control and Optimization, Vol. 48 (2010), pp. 5276-5293.

[30] J.G. Dai, D. Yao, "Brownian Inventory Models with Convex Holding Cost, Part 1: AverageOptimal Controls", Stochastic Systems, Vol. 3 (2013), Issue 2, pp. 442-499.

[31] J.G. Dai, D. Yao, "Brownian Inventory Models with Convex Holding Cost, Part 2: DiscountOptimal Controls" (2013), Stochastic Systems, Vol. 3, Issue 2, pp. 500-573.

[32] T. De Angelis and G. Ferrari, "A Stochastic Partially Reversible Investment Problem on a Finite Time-Horizon: Free-Boundary Analysis", Stochastic Processes and their Applications Vol. 124(3) (2014), pp. 4080-4119.

[33] T. De Angelis, S. Federico and G. Ferrari, "Optimal Boundary Surface for Irreversible Investment with Stochastic Costs", Mathematics of Operations Research, Vol. 42, No. 4, pp. 11351161 (2017).

[34] J.F. Eastham, K.J. Hastings "Optimal Impulse Control of Portfolios", Mathematics of Operations Research, Vol. 13 (1988), Issue 4, pp. 588-605.

[35] M. Egami, "A direct solution method for stochastic impulse control problems of onedimensional diffusions", SIAM Journal on Control and Optimization, Vol. 47, No. 3, pp. 1191$1218,2008$.

[36] L. Evans, Partial Differential Equations (Second Edition), Graduate Studies in Mathematics, Vol. 19, AMS Providence, 2010.

[37] S. Federico, H. Pham, "Characterization of optimal boundaries in reversible investment problems”. SIAM Journal on Control and Optimization, Vol. 52, No. 4, pp. 2180-2223 (2014).

[38] G. Ferrari, "On an Integral Equation for the Free-Boundary of Stochastic, Irreversible Investment Problems", The Annals of Applied Probability, Vol. 25(1) (2015), pp. 150-176.

[39] G. Ferrari, T. Koch. "On a Strategic Model of Pollution Control”, Annals of Operations Research (2018). (DOI) 10.1007/s10479-018-2935-7

[40] G. Ferrari, P. Salminen, "Irreversible Investment under Lèvy Uncertainty: an Equation for the Optimal Boundary, Advances in Applied Probability 48(1) (2016)”, pp. 298-314.

[41] X. Guo and H. Pham, "Optimal partially reversible investments with entry decision and general production function", Stochastic Processes and their Applications, Vol. 115 (2005), No. 5, pp. 705-736.

[42] J.W. Gu, M. Steffensen, H. Zheng, "Optimal Dividend Strategies of Two Collaborating Businesses in the Diffusion Approximation Model", Mathematics of Operations Research, Vol. 43,pp. 377-398, 2018.

[43] X. Guo and G. Wu, "Smooth fit principle for impulse control of multidimensional diffusion processes”, SIAM Journal on Control and Optimization, Vol. 48, No. 2, pp. 594-617, 2009.

[44] J.M. Harrison, T.M. Sellke, A.J. Taylor, "Impulse Control of Brownian Motion”, Mathematics of Operations Research, Vol. 8 (1983), Issue 3, pp. 454-466. 
[45] S. He, D. Yao, H. Zhang, "Optimal Ordering Policy for Inventory Systems with QuantityDependent Setup Costs", Mathematics of Operations Research, Vol. 42 (2017), Issue 4, pp. 979-1006.

[46] K.L. Helmes, R.H. Stockbridge, and C. Zhu, "A Measure Approach for Continuous Inventory Models: Discounted Cost Criterion", SIAM Journal on Control and Optimization Vol. 53 (2015), No. 4, pp. 2100-2140.

[47] J.E. Hodder and A. Triantis, "Valuing flexibility as a complex option", Journal of Finance, Vol. 45 pp. 549-565, 1990.

[48] I. Karatzas and S.E. Shreve, Brownian Motion and Stochastic Calculus. Springer, $2^{\text {nd }}$ edition, 1991.

[49] R. Korn, "Portfolio Optimization with strictly positive transaction costs and impulse control", Finance and Stochastics, Vol. 2, pp. 85-114, 1998.

[50] A. Jack and M. Zervos, "Impulse Control of One-dimensional Itô Diffusions with an Expected and a Pathwise Ergodic Criterion", Applied Mathematics and Optimization, Vol. 54, pp.7193, 2006.

[51] M. Jeanblanc-Picqué, "Impulse control method and exchange rate", Mathematical Finance, Vol. 3, Issue 2, pp. 161-177, 1993.

[52] R. McDonald and D. Siegel, "The Value of Waiting to Invest" The Quarterly Journal of Economics, Vol. 101, Issue 4, pp. 707-727, 1986

[53] A.S. Manne, "Capacity expansion and probabilistic growth", Econometrica, Vol. 29 (1961), No. 4, pp. 632-649.

[54] D.C. Mauer and A. Triantis, "Interactions of corporate financing and investment decisions: a dynamic framework", Journal of Finance, Vol. 49, pp. 1253-1277, 1994.

[55] A. Merhi and M. Zervos, "A model for reversible investment capacity expansion", SIAM Journal on Control and Optimization, Vol. 46 (2007), No. 3, pp. 839-876.

[56] D. Mitchell, H. Feng, K. Muthuraman, "Impulse control of interest rates", Operations Research, Vol. 62 (2014), Issue 3, pp. 602-615.

[57] J. Morton and B. Oksendal, "Optimal portfolio management with fixed costs of transactions", Mathematical Finance, Vol. 5, pp. 337-356, 1995.

[58] K. Muthuraman, S. Seshadri, Q. Wu, "Inventory Management with Stochastic Lead Times", Mathematics of Operations Research, Vol. 40 (2014), Issue 2, pp. 302-327.

[59] A. Øksendal, "Irreversible investment problems", Finance and Stochastics, Vol. 4 (2000), No. 2, pp. 223-250.

[60] B. Øksendal, A. Sulem, Applied Stochastic Control of Jump-Diffusions, Springer-Verlag (2007). 
[61] B. Øksendal, J. Ubøe, T. Zhang, "Non-robustness of some impulse control problems with respect to intervention costs", Stochastic Analysis and Applications Vol. 20 (2002), No. 5, pp. 999-1026.

[62] M. Ormeci, J.G. Dai, J. Vande Vate, "Impulse Control of Brownian Motion: The Constrained Average Cost Case", Mathematics of Operations Research, Vol. 56 (2008), Issue 3, pp. 618629.

[63] H. Pham, Continuous-time stochastic control and applications with financial applications, Springer, Series Stochastic Modelling and Applied Probability, Vol. 61 (2009).

[64] F. Riedel and X. Su, "On irreversible investment", Finance and Stochastics, Vol. 15 (2011), No. 4, pp. 607-633.

[65] R.T. Rockafellar, Convex Analysis, Princeton University Press (1970).

[66] P. Salminen, B.Q. Ta, "Differentiability of excessive functions of one-dimensional diffusions and the principle of smooth-fit". Advances in Mathematics of Finance, Vol. 104 (2015), pp. 181-199.

[67] H. Scarf, "The optimality of $(S, s)$ policies in the Dynamic Inventory Problem", in Mathematical Methods of Social Sciences 1959: Proceedings of the First Stanford Symposium, pp. 196-202, S. Karlin and P. Suppes (eds.), Stanford University Press (1960).

[68] A. Sulem, "A solvable one-dimensional model of a diffusion inventory system", Mathematics of Operations research, Vol. 11 (1986), pp. 125-133.

[69] A. Sulem, "Explicit Solution of a Two-Dimensional Deterministic Inventory Problem", Mathematics of Operations Research (1986), Vol. 11, Issue 1, pp. 134-146.

[70] H. Wang, "Capacity expansion with exponential jump diffusion process", Stochastics and Stochastics Reports, Vol. 75 (2003), No. 4, pp. 259-274.

[71] K. Yamazaki. "Inventory Control for Spectrally Positive Lévy Demand Processes", Mathematics of Operations Research, Vol. 42 (2016), Issue 1, pp. 302-327.

[72] J. Yong and X.Y. Zhou, Stochastic Controls: Hamiltonian Systems and HJB equations, Springer-Verlag, Berlin, New York, 1999. 